\title{
Capillarity and phase-mobility of a hydrocarbon gas-liquid system
}

Ying Gao ${ }^{1}$, Apostolos Georgiadis ${ }^{1}$, Niels Brussee ${ }^{1}$, Ab Coorn $^{1}$, Hilbert van der Linde ${ }^{1}$, Jesse Dietderich ${ }^{2}$, Faruk Omer Alpak ${ }^{2}$, Daniel Eriksen ${ }^{1}$, Miranda Mooijer-van den Heuvel ${ }^{1}$, Matthias Appel ${ }^{1}$, Tibi Sorop ${ }^{1}$, Ove Bjørn Wilson ${ }^{1, a}$, and Steffen Berg ${ }^{1, *}$

${ }^{1}$ Shell Global Solutions International B.V., Grasweg 31, 1031 KC Amsterdam, The Netherlands

${ }^{2}$ Shell International Exploration and Production Inc., 3333 Hwy 6, Houston, 77082 TX, USA

Received: 19 February 2021 / Accepted: 19 April 2021

\begin{abstract}
When oil fields fall during their lifetime below the bubble point gas comes out of solution. The key questions are at which saturation the gas becomes mobile ("critical gas saturation") and what the gas mobility is, because mobile gas reduces the production of oil significantly. The traditional view is that the gas phase becomes mobile once gas bubbles grow or expand to a size where they connect and form a percolating path. For typical 3D porous media the saturation corresponding to this percolation limit is on the order of $20 \%$. However, significant literature report on gas mobility below lower limits of percolation thresholds i.e. below $0.1 \%$. A direct experimental insight for that is lacking because laboratory measurements are notoriously difficult since the formation of gas bubbles below the bubble point includes thermodynamic and kinetic aspects, and the pressure decline rates achievable in laboratory experiments are orders of magnitude higher than the decline rates in the field. Here we study the nucleation and transport of gas coming out of solution in-situ in 3D rock using X-ray computed micro tomography which allows direct visualization of the nucleation kinetics and connectivity of gas. We use either propane or a propane-decane mixture as model system and conduct pressure depletion in absence of flow finding that - consistent with the literature - observation of the bubble point in the porous medium is decreased and becomes pressure decline rate dependent because of the bubble nucleation kinetics. That occurs in single-component systems and in hydrocarbon mixtures. Pressure depletion in absence of flow results in critical gas saturations between 20 and $30 \%$ which is consistent with typical percolation thresholds in 3D porous structures. That does not explain experimentally observed critical gas saturations significantly below $20 \%$. Also, the respective pore level fluid occupancy where pores are filled with either gas or liquid phase but not partially with both as in normal 2-phase immiscible systems rather diminishes connectivity of gas and liquid phases. This observation indicates that likely other mechanisms play a role in establishing gas mobility at saturations significantly below 20\%. Experiments under flow conditions, where gas is injected near the bubble point suggest that diffusion may significantly contribute to the transport of gas and may even be the dominant transport mechanism at field relevant flow rates. The consequence of diffusive transport are compositional gradients where locally the composition is such gas nucleation may occur. That would lead to a disconnected but mobile gas distribution ahead of the convective front. Furthermore, diffusive exchange leads to ripening and anti-ripening effects which influences the distribution for which we see evidence in pressure depletion experiments but not so much at low rate gas injection. Respective relative permeability computed from the imaged fluid distributions using a lattice Boltzmann approach show distinctly different behavior between pressure depletion and flowing conditions. These findings suggest that capillarity in a gasliquid hydrocarbon mixture is far more complex than in a 2-phase immiscible system. Capillarity is coupled to phase behavior thermodynamics and kinetics on a fast time scale and diffusion-dominated mechanisms such as ripening and anti-ripening effects at a slow time scale. While the consequences for the current experimental and field modelling approaches are not yet fully clear, this shows that more research is needed to fully understand these effects and their implications.
\end{abstract}

\footnotetext{
* Corresponding author: steffen. berg@shell.com

${ }^{a}$ Now at Petroleum Development Oman.
} 


\section{Introduction}

The critical gas saturation marks the onset at which gas, that comes out of solution at or below the bubble point pressure [1] of a live crude oil, becomes mobile in reservoir rock. It is quite often an important parameter for the production of oil fields, which typically during their lifetime fall below the bubble point pressure as for gas saturations higher than the critical gas saturation the oil production may be significantly reduced (a simplistic sketch is shown in Fig. 1).

There have been many laboratory experiments that attempted to determine the critical gas saturation and values in the range of $0.5-50 \%$ have been reported, depending on fluid composition and experimental protocols [2-14], although the majority of publications gives a critical gas saturation below $5 \%$ [2]. In laboratory experiments it is very difficult to reproduce the conditions in the field because the pressure decline rates in the field are typically orders of magnitude smaller than those achievable in the laboratory [2] and respective values of the critical gas saturation observed in the laboratory in general have to be extrapolated to the pressure decline rates in the field [2-8]. The reasoning is that the formation of gas bubbles is a nucleation process with a complicated interplay between pressure decline rate, supersaturation of the liquid, the rate of nucleation and growth kinetics of the gas bubbles [2, 5, 7, 15]. If the pressure decline rate is too high, bubble nucleation and growth could be suppressed. Extrapolation to field pressure-decline rates as displayed in Figure 2A is believed to be a permissible methodology. However, critical gas saturations obtained in this way can be as low as $0.5 \%$, therefore significantly below the percolation threshold of $3 \mathrm{D}$ structures, which is roughly in the range of $20-32 \%$ [16-20] which are also values suggested by authors for pressure depletion mode experiments without flow [21, 22]. This suggests that another source of uncertainty is the underlying flow regime [5]. The volume expansion of the growing gas bubbles may, at larger scale, create flow similar to the solution gas drive $[1,23]$. Laboratory experiments where the critical gas saturation is probed by a flow measurement i.e. when the first gas is produced observe typically a much lower critical gas saturation than in constant rate depletion experiments [2]. The very low viscosity of the gas could cause viscous fingering even at pore scale [24] which could be a potential explanation for a connected pathway below a 3D isotropic percolation threshold as sketched in Figure 2B. Largely reduced interfacial tension near the critical point may further favor such a process [25, 26]. Laboratory experiments on air sparging also indicated mobility of gas without a permanent connected path [21, 27-29]. In water-oil immiscible displacement similar processes have been observed, which are termed "ganglion dynamics" $[30,31]$ sketched in Figure 2C. It is upfront not clear to which extent such processes could play a role in the correct estimate of the onset of gas mobility in field operations. It is, however, clear that the problem is more complex than previously believed and that a more detailed understanding of the pore scale fluid distribution is required to develop an intuitive understanding, and a respective methodology for quantitative assessment.
Darcy-scale/core flood experiments are not particularly suitable for achieving that. Even though saturation monitoring $[8,9,15]$ has improved the interpretation, critical gas saturations are indirectly inferred and would still need to be extrapolated. Moreover, flow experiments often employ (very) high velocity and saturation gradients much higher than in the field $[8,9]$. That makes it difficult to identify the underlying flow regime and distinguish quasi-static bubble nucleation from unstable flow and gas mobility in non-percolating settings.

In this study we use X-ray computed micro tomography to directly image the pore scale distribution of gas and assess whether the critical gas saturation coincides with the percolation threshold. We chose a hydrocarbon gasliquid model system to provide a proof of concept. We investigate systematically, starting with a single-component system, how the phase transition from liquid to gas state manifests itself in-situ in the porous medium with respect of the pore level distribution of gas and liquid. We then extend the study to a two-component model system (propane-decane). We explore the flow regimes of constant rate pressure depletion and injection of gas. Relative permeability is computed from the imaged pore scale fluid distributions. We find that, in addition to the bubble nucleation and growth, and convective flow, also the diffusive transport can be of significant importance, which is an aspect that has been so far overlooked in the previous studies.

\section{Methods and materials}

\subsection{Experimental setup}

The heart of the experimental setup is micro-core of porous rock (Bentheimer outcrop sandstone, porosity $=19.1 \%$, permeability is $2500 \mathrm{mD}$ ) with $4 \mathrm{~mm}$ diameter and $20 \mathrm{~mm}$ length (which is strongly hydrophilic as it has not been exposed to crude oil, and cleaned with isopropanol), mounted in a PEEK core holder that can be pressurized and temperature controlled (up to 30 bar and $60{ }^{\circ} \mathrm{C}$ ). Fluids are injected and produced using high pressure syringe pumps (Quizix), whilst imaging the fluid distribution in the pore space is done by X-ray computed micro-tomography

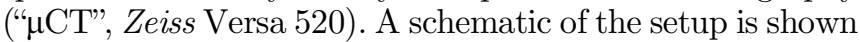
in Figure 3A. More details are given in [32, 33].

\subsection{Fluids}

As this is a proof-of-concept study, either n-propane or a mixture of n-decane and n-propane were chosen as gasliquid model systems, with an extended two-phase region within the operating envelope of the setup shown in Figure 4 . The respective phase behavior which has been computed using the Shell Modified Redlich-Kwong (SMIRK) Equation Of State (EOS) [34] is displayed in Figure 4 . For a $50 \%$ molar fraction of propane, at the temperature inside the $\mu \mathrm{CT}$ of $27^{\circ} \mathrm{C}$ (the temperature in the pumps was $22^{\circ} \mathrm{C}$ ) a bubble point is expected around 4.7-4.8 bar, at an interfacial tension of $17 \mathrm{mN} / \mathrm{m}$. For the experimental uncertainties in terms of achieving the desired 


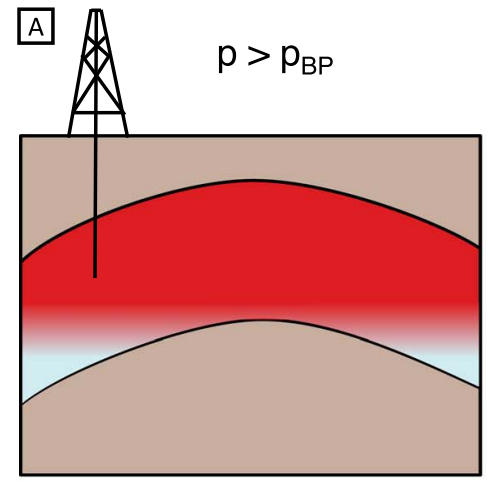

Producing oil

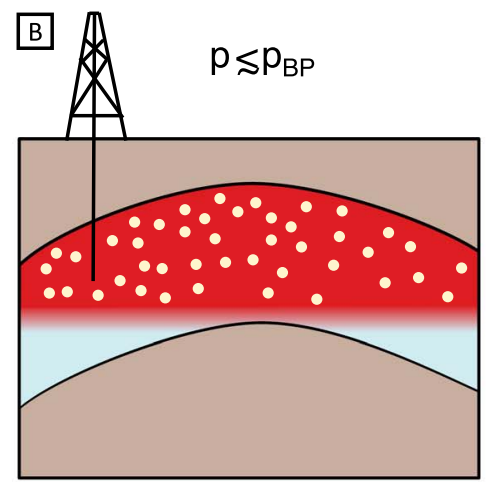

Producing mainly oil

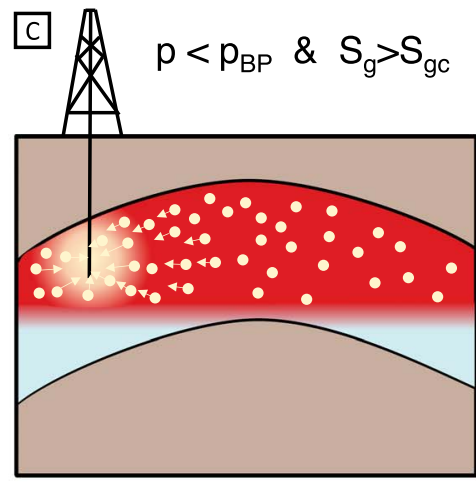

Producing mainly gas

Fig. 1. Production characteristics of an oil field that is initially above the bubble point pressure $p>p_{\mathrm{BP}}(\mathrm{A})$ and eventually pressure declines below the bubble point pressure $p \lesssim p_{\mathrm{PB}}(\mathrm{B})$. When the critical gas saturation is reached $S_{g}>S_{g, \mathrm{c}}(\mathrm{C})$, gas becomes mobile and due to the large viscosity ratio excessive amount of gas is produced.

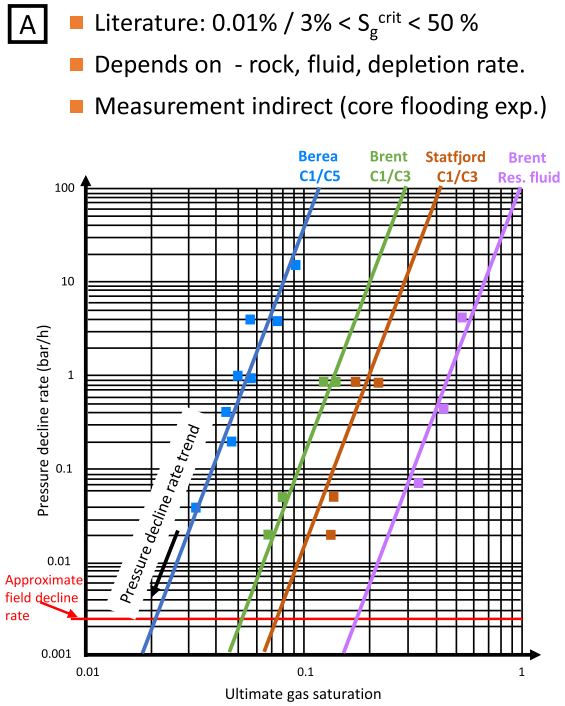

\section{B Fluid phase mobility for 2-phase immiscible systems \\ - Unstable displacement (pore scale viscous fingering): Connected but below percolation threshold}

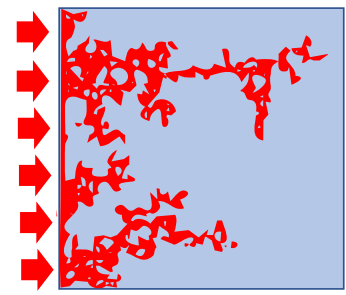

C

- Ganglion dynamics: disconnected by mobile

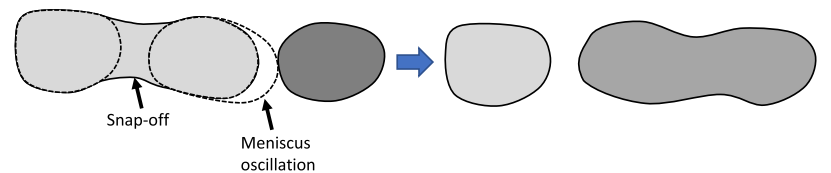

Fig. 2. Extrapolation of the gas saturation from the pressure decline rate in the laboratory to field conditions (A, adapted from [2]) and established mechanisms for mobility for 2-phase immiscible displacement where unstable displacement at the pore scale can lead to connectivity below the percolation threshold (B, adapted from [24]) and ganglion dynamics providing phase mobility without permanent connectivity (C, adapted from [44]).

mole fraction of $50 \%$ and reaching thermal equilibration a bubble point between 2 and 5 bar is expected.

Without any water being present in the experiments, the liquid hydrocarbon phase is always the wetting phase covering the rock surface while the gas is the non-wetting phase and would therefore accumulate in the center of pores [35], as observed in the experiments (see Figs. 5, 11 and 12). Throughout the experiments, the wettability stays approximately constant, i.e. the liquid-phase remains fully coating the solid surfaces.

\subsection{Image processing}

During pressure depletion and/or gas injection, the liquid and gas distributions in the rock sample are imaged (at pressure equilibrium conditions) by X-ray computed micro-tomography using a Zeiss Versa 520 micro-CT scanner. The following scan protocol was optimized for shortest possible scan time while maintaining sufficient resolution and image quality to segment individual gas bubbles: $0.4 \times$ magnification, $80 \mathrm{kV}$ at $7 \mathrm{~W}$ power, applying a $2 \times 2$ binning on the detector at an exposure time of $1.3 \mathrm{~s}$, with 1401 projections, which results in a time for each scan of about 1 h. First a scan of the dry rock ("dry scan") was taken with higher quality settings, and segmented into pore space and grains, using nonlocal means filtering [36] and watershed segmentation using Avizo 9 (ThermoFisher). All subsequent scans with fluids present in the pore space were first registered onto the dry scan, the pore space masked by the segmented dry scan and gas and liquid in 

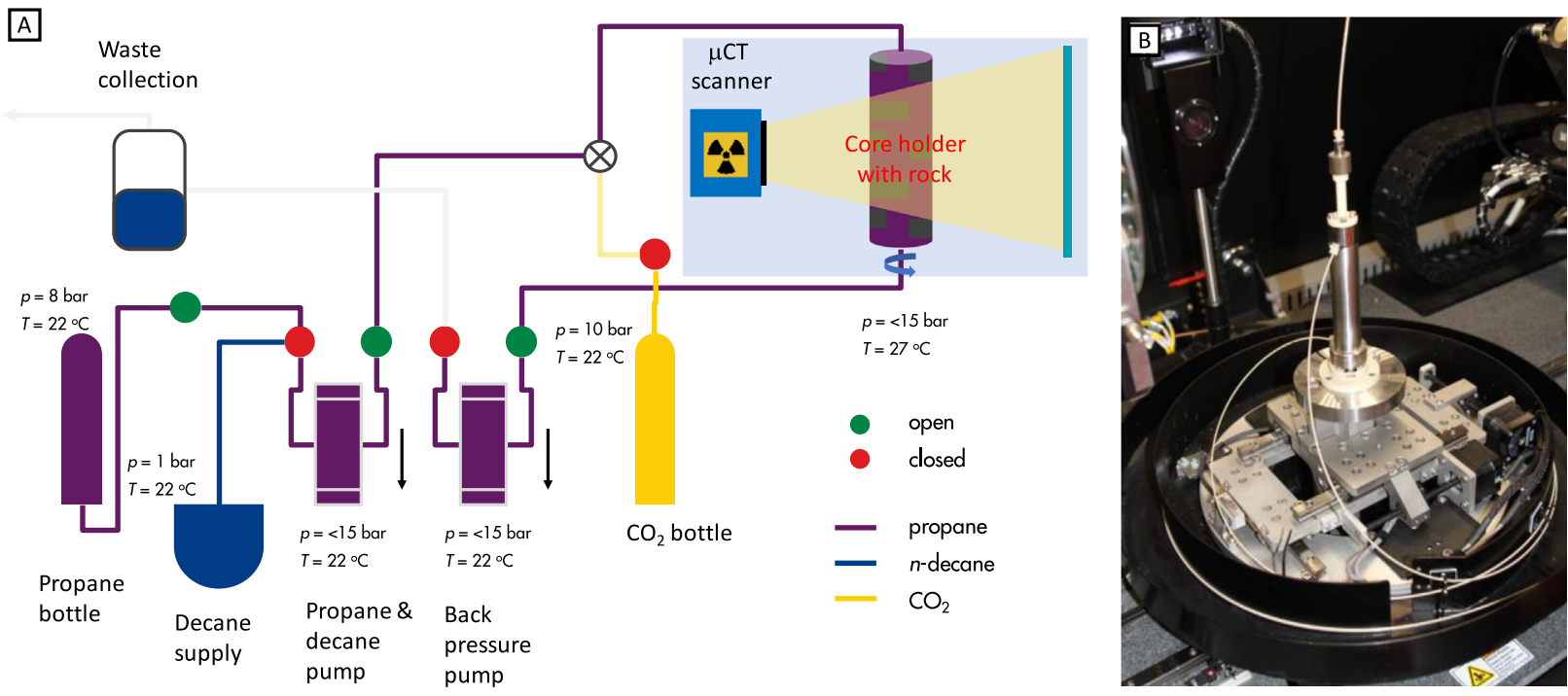

Fig. 3. Schematic drawing of the setup which includes flow loop (A) and core holder in the micro-CT scanner (B).
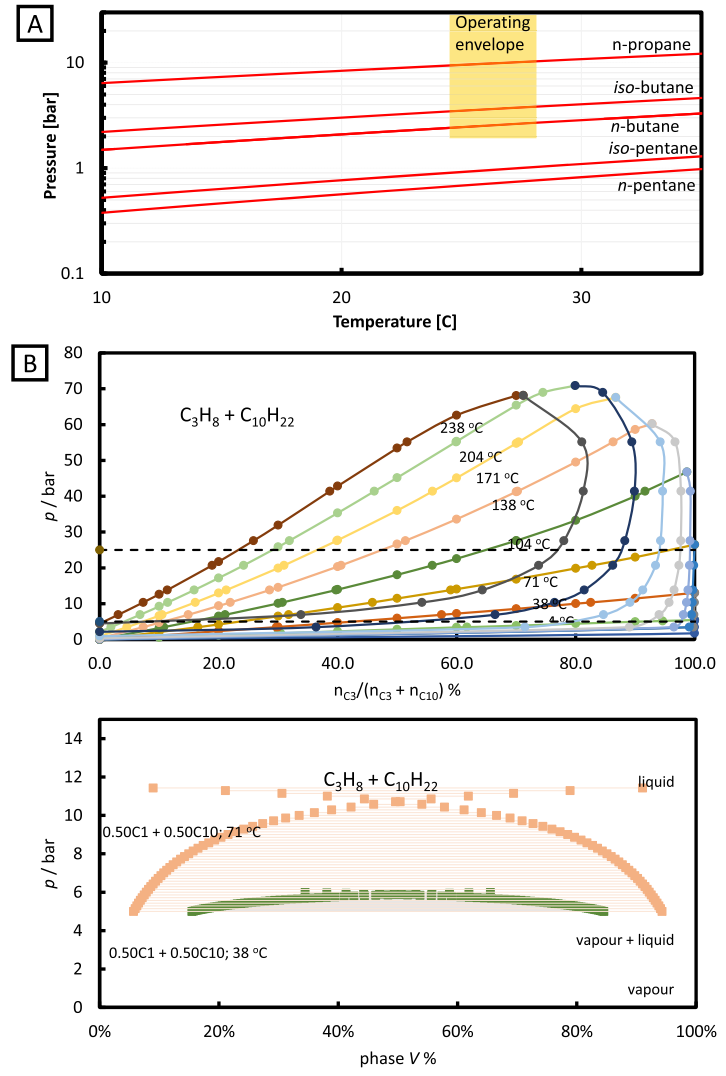

Fig. 4. Phase behavior of several pure components including n-propane (A) and the propane-decane system (B) in the pressure-temperature range corresponding to the operating envelope of the micro-CT flow cell (dashed black lines, left), and the respective phase split computed with SMIRK EOS [34].

the pore space segmented by using the watershed method. For the gas phase a cluster analysis was applied where each individual gas cluster was labelled and visualized in a different color. An example of the workflow is shown in Figure 5.

This workflow for the model system can be also applied to other hydrocarbon systems and proves that there is sufficient X-ray contrast between gas and liquid phases such that no contrast agents are required and, therefore, avoid any potential impact on the fluid properties.

\subsection{Flow simulations}

In this work, the 3D spatial distribution of liquid and gas phases from the segmented micro-CT scans were used to compute (connected pathway) relative permeability following a similar methodology as in [37], using a MultiRelaxation-Time Lattice Boltzmann (MRT-LBM) method implemented to run on high-performance general purpose GPU (GPGPU) computing clusters by use of a novel distributed parallel programming paradigm. Note that the phase change is not modelled by LBM but taken from the experiment (segmented micro-CT images). The connected pathway relative permeability computation protocol serves here as a practical computationally effective solution to an otherwise computationally costlier multi-phase simulation alternatives, see e.g. [38]. Crucially, it is also a physically suitable approach, since for the gas-liquid scenario where gas is a completely non-wetting phase, the connected pathway contribution to relative permeability dominates over the flux contribution of ganglion dynamics which can be therefore neglected [30, 31].

The MRT-LBM method used in this work is based on the MRT model and a precise treatment of body force that drives the flow [37]. The Boltzmann equation is discretized in space, velocity (momentum), and time coordinates using a 3D 19-velocity grid (D3Q19 scheme), which provides the optimal compromise between accuracy and computational efficiency. The conventional approach of representing particle collision (or relaxation towards an equilibrium state due to collision) is based on the single-relaxation time 

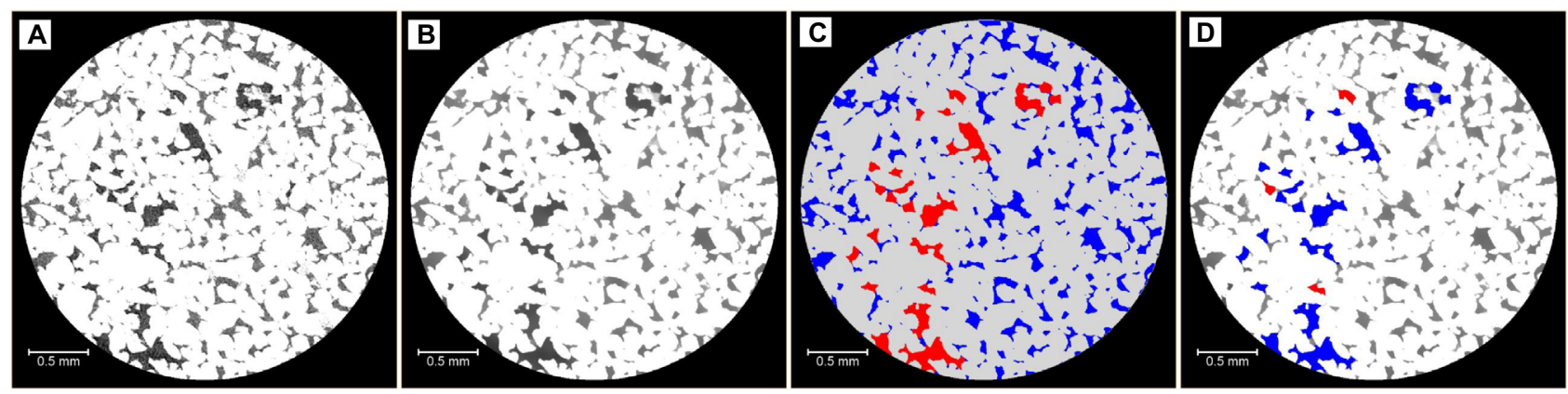

Fig. 5. Slice of a typical micro-CT scan. Raw data (A), filtering and contrast enhancement (B), after segmentation (red $=$ gas, blue $=$ liquid) (C) and after cluster analysis (now red and blue are different gas clusters) (D). Note that the color scheme used in the segmented image before the cluster analysis denotes rock, fluid and gas, whereas the color scheme after the cluster analysis (D) is used to indicate individual gas clusters.

Bhatnagar-Gross-Krook (BGK) model [39], where a single relaxation time is employed to describe the relaxation processes. However, this model has several deficiencies, such as numerical instabilities at relatively high Reynolds numbers [37]. In the MRT model [40], the velocity distribution function is transformed into moment space, where individual moments can be relaxed at individual rates. Overall, the MRT model has several advantages over the conventional single-relaxation time BGK model, and those include: (1) enhanced numerical stability, (2) independent bulk and shear viscosities, and (3) viscosity-independent, non-slip boundary conditions [37]. The drawback of the MRT model is that it is slightly more computationally demanding compared to the BGK model. This minor hurdle is easily overcome through a GPGPU implementation of the MRT model.

In MRT-LBM, fluid is driven by a body-force term by imposing a loop boundary condition on the inlet and outlet faces of the domain. The same body force is used for the fluid that leaves the domain at a given domain-boundary surface (e.g., the outlet face of the domain) and enters the domain at the opposite boundary surface (e.g., the inlet face of the domain). A precise treatment of body-force is required to eliminate errors in velocity gradients. For the velocity vector, the zero-slip boundary condition is imposed on the surfaces between rock grains and fluid within the pore space. The mathematical formulation and code implementation details can be found in [37].

\section{Results}

Starting point is the assessment of the general behavior of an in-situ phase transition from liquid to gas inside of a porous medium. We begin with pressure depletion experiments of the pure component system n-propane and then extend to the two-component propane-decane system. In order to cover the range of possible flow regime, for the two-component case, two classes of experiments have been conducted: (1) pressure depletion and re-pressurization without external flow and (2) gas injection near the bubble point.

\subsection{Pressure depletion experiments with single-component system (propane)}

At pressures above the bubble point the pore space was entirely filled with liquid propane. When decreasing the pressure, at the propane bulk bubble point (of 10.8 bar) there is no formation of gas bubbles detected. Only when decreasing the pressure more than 0.8 bar below the bubble point pressure, the gas bubbles form as shown in Figure 6 .

The respective micro-CT scans reveal that at pressures below the bulk phase bubble point $(P<10.8$ bar $)$ but above 10.0 bar where the bubble nucleation occurs, there is only liquid propane in the pore space and no gas. At the point of bubble nucleation we find that all liquid is either displaced out of the pore space or converted into gas, which is logical because the thermodynamic co-existence between liquid and vapor phase is only at the bubble point which is exceeded significantly when the gas bubbles nucleate, i.e. we are dealing with a super-heated liquid.

The nucleation of gas bubbles is associated with a large volume expansion which is recorded by the back-pressure pump cylinder. The servo control system of the pump is configured to adjust the pump cylinder such that a constant pressure is maintained. Here, because of the volume expansion, the cylinder is retracted. Depending on the exact conditions the gas bubble expansion of the super-heated liquid can occur so rapid that the pump cylinder cannot retract fast enough which is also creating problems for the servo control system of the pump and the pressure cannot be kept constant anymore. The consequence may be a pressure overshoot as seen in Figure 6A or even an oscillatory behavior of pressure as in a second experiment shown in Figure 7A. While in the pressure-time domain the oscillation may not appear that significant, in the pressure-volume domain it forms significant hysteresis cycles as shown in Figure 7B. Also note that the exact pressure for nucleation can vary from experiment to experiment as nucleation is an inherently unstable process that occurs over a range of 


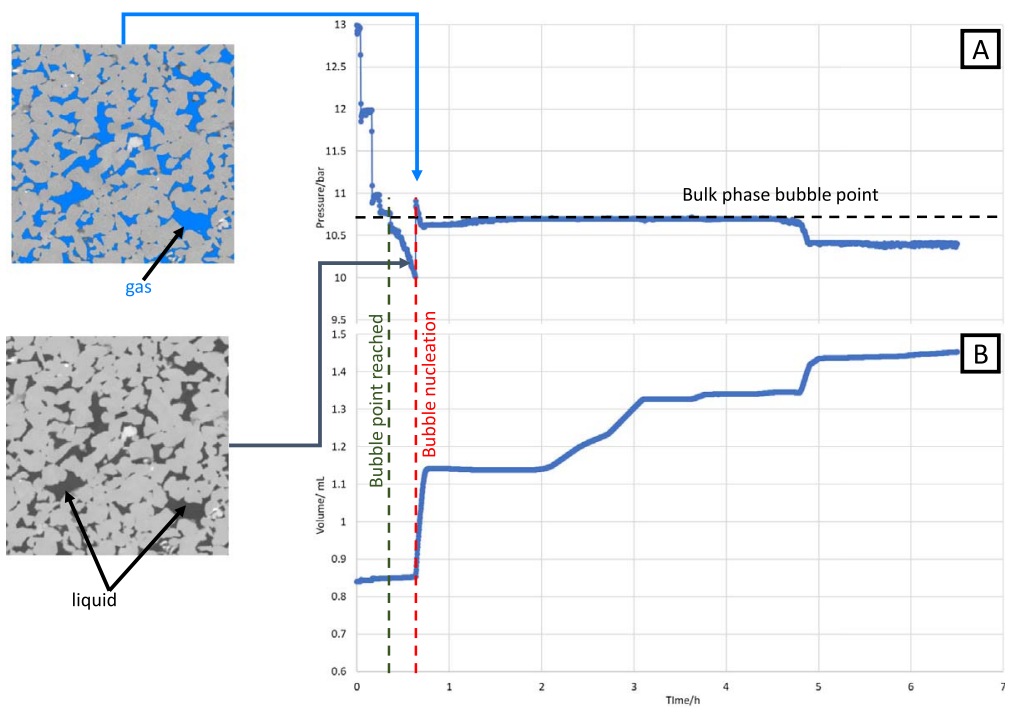

Fig. 6. Pressure (A) and pump volume (B) during a pressure depletion experiment with n-propane. The bulk phase bubble point is crossed at about $t \sim 0.4 \mathrm{~h}$ but no gas nucleates yet. Only after reducing the pressure further, at $t \sim 0.7 \mathrm{~h}$ gas nucleates in the pore space displacing practically all liquid from the pore space in the field of view as shown by the $\mu \mathrm{CT}$ cross sections on the right.
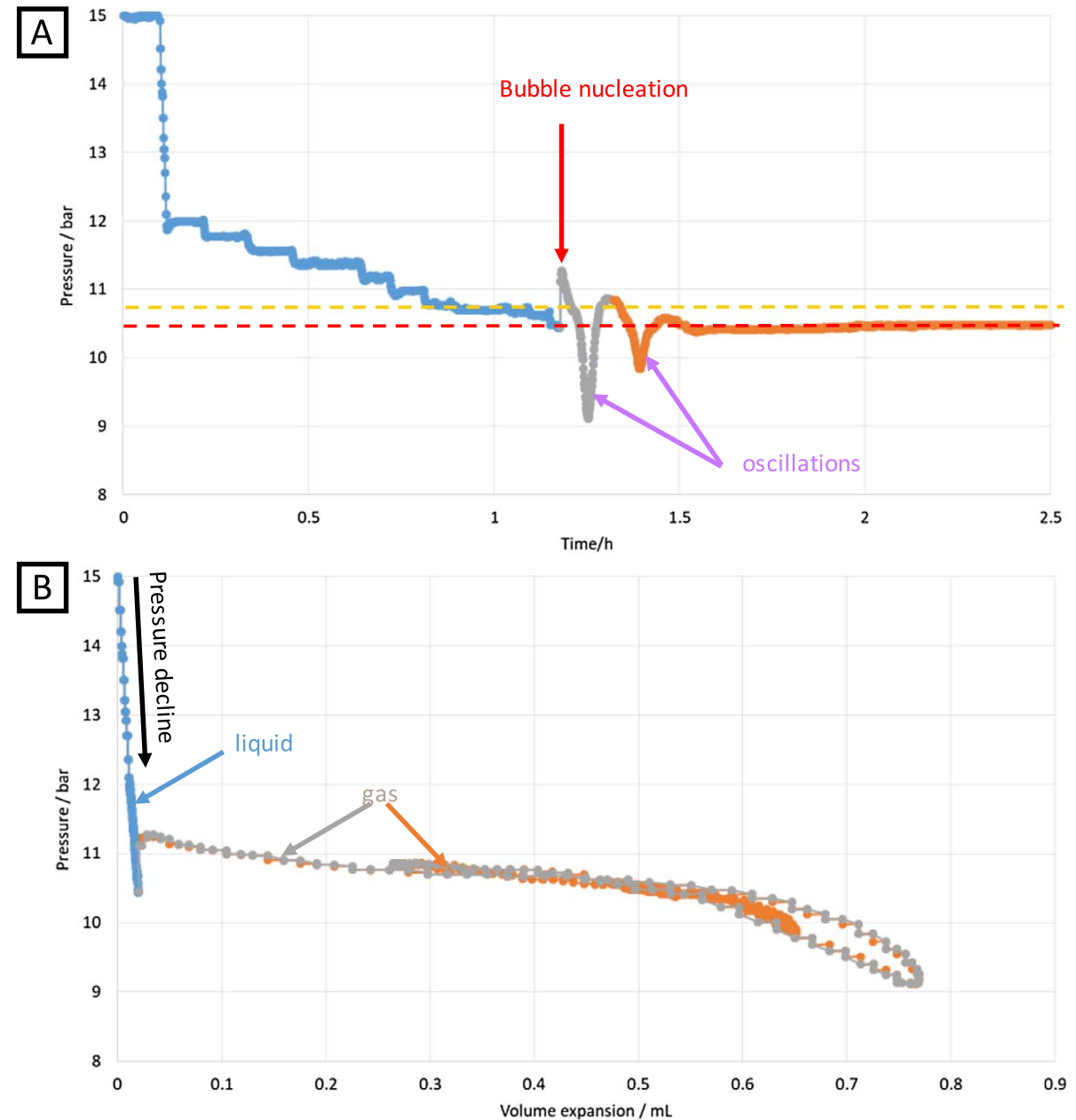

Fig. 7. Pressure vs. time (A) and pressure vs. volume (B) for a second pressure-depletion experiment with n-propane. When gas bubbles are nucleated, the volume expansion is too rapid for the back-pressure pump to follow (mechanically and for the servo control systems) which causes pressure oscillations. These are significant in the pressure-volume domain. 

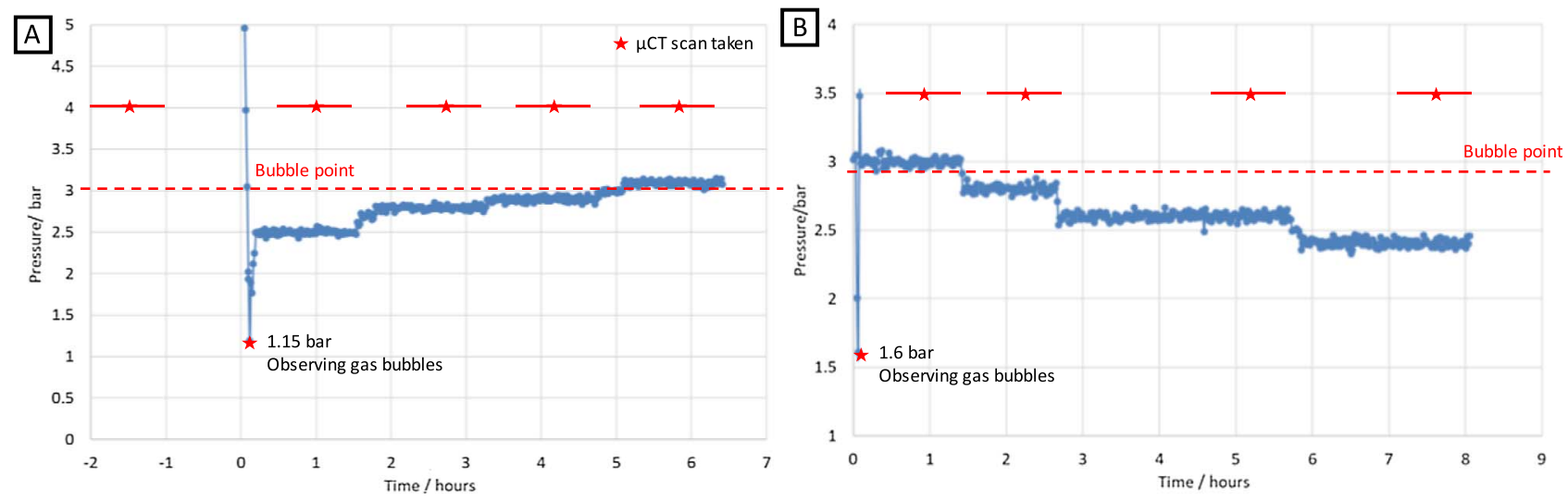

Fig. 8. Pressure vs. time for a pressure depletion experiment in a 50:50 propane-decane mixture and subsequent re-pressurization to the bubble point (A). In order to develop a protocol for a more controlled bubble nucleation, a quick pressure drawdown can be used to nucleate bubbles (B) which are then expanded in a more controlled way by quickly re-pressurizing to approximately the bubble point and then slowly decreasing the pressure.

pressure and temperatures but not always at the exactly identical one (compare Figs. 6 and 7 which are repeat experiments at otherwise identical conditions but the pressure at which nucleation occurs is slightly different).

From the behavior illustrated in Figures 6 and 7 it is clear that the single-component system may not be a good model system for representing the behavior of reservoir conditions because the 2-phase envelope is too narrow and the gas nucleation too uncontrollable. On the other hand, the two-component propane-decane system may be more suitable because it has a much wider 2-phase envelope (Fig. 4) and is expected that after gas bubble nucleation not all liquid is immediately displaced from the pore space.

Nevertheless, the single-component system does establish some of the conceptual difficulties of performing experiments on gas nucleation processes, as it always is a somewhat uncontrolled process which occurs in general not at the bulk thermodynamic condition. The underlying reason is that the bulk phase thermodynamic bubble point is in fact a phase coexistence condition of pre-formed phases but does not describe the phase transition itself. Depending on the phase separation mechanism, the difference between the thermodynamic and the observed bubble point can be caused by thermodynamic (e.g. difference between binodal and spinodal) and kinetic effects/reasons. Bubble formation requires in addition to thermodynamic condition in terms of pressure and temperature also a nucleation seed. Nucleation can be either homogeneous i.e. caused by thermal fluctuations in the bulk phase, or heterogeneous i.e. at the rock surface. Since in our experiments i.e. in absence of water gas is always the least wetting phase, it is uncertain that in our experiments nucleation is homogeneous. Since nucleation takes time, if the pressure decline rate is faster than the nucleation and growth rates, the system will always be in a non-equilibrium (kinetic effects) resulting in an over-heated or super-saturated liquid. In case of homogeneous nucleation, while in a large PVT cell there is sufficient chance for a nucleation seed to be formed by fluctuations within the liquid, in pores inside the rocks the probability of that to happen is smaller. That leads to a liquid supersaturated in dissolved gas which comes out of solution at once when a nucleation eventually occurs. This phenomenon has been already extensively described in the literature [5] and it is the root cause for the difficulties in constant pressure depletion experiments to reach a true bubble point.

\subsection{Pressure depletion experiments in a 2-component system (decane-propane)}

Even though the 2-component propane-decane system has a much wider phase envelope (Fig. 4), when depleting the pressure starting from approximately 15 bars, just like the pure propane case we observe the depression of the bubble point and unstable bubble nucleation (see Fig. 8A).

As shown in the micro-CT scans in Figure 9, a key difference between the single-component (propane) and the two-component (decane-propane) systems is that for decane-propane after the nucleation of gas bubbles some liquid remains in the pore space. In order to develop a protocol for a more controlled bubble nucleation, a quick pressure drawdown (Fig. 8B) can be used to nucleate bubbles which are then expanded in a more controlled way by quickly re-pressurizing to approximately the bubble point and then slowly decreasing the pressure.

In this work we followed two strategies to address the supersaturation issue, which hinges on the suppression of bubble nucleation. The key of both strategies is to start with a situation where gas bubbles are already present. The first strategy is to conduct experiments in a re-pressurization mode, i.e. starting from a situation far below the bubble point pressure where gas bubbles have been nucleated. The second strategy is to first nucleate bubbles in a quick pressure drawdown below the bubble point pressure but then immediately to return to a pressure slightly above the bubble point and then deplete pressure in 

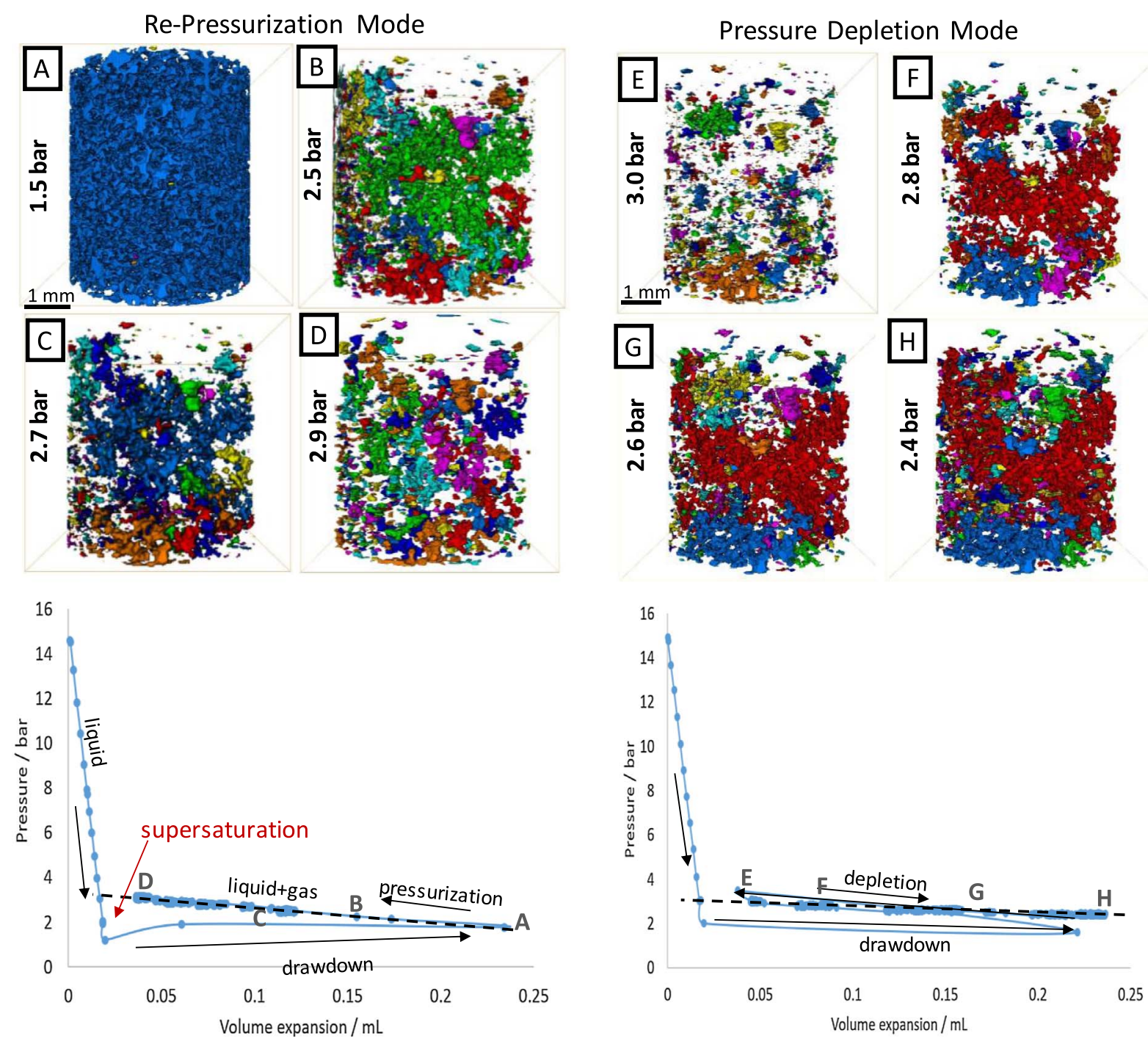

Fig. 9. Micro-CT images (top panel) where colors indicate individual gas clusters, and respective pressure-volume curves (bottom panel) for the constant rate depletion experiments. Constant rate depletion leads to supersaturation where eventually gas nucleates which leads to instantaneous percolation. Upon re-pressurization eventually the percolation threshold is reached (left column). A quick drawdown below the bubble point leads to nucleation of gas bubbles, which - after immediate pressure increase near the bubble point - grow successively until they connect and in this way the percolation threshold i.e. the critical gas saturation is reached (right column) [32].

small steps and thereby slowly growing the gas bubbles until percolation threshold is passed.

The two strategies displayed in Figures 8A and 8B, i.e. re-pressurization to reach the bubble point and quick draw-down followed by controlled pressure depletion, are shown in Figure 9 (on the left and the right) in more detail. The re-pressurization mode starts with a large initial gas volume that percolates from top-to-bottom (Fig. 9A). Increase in pressure successively leads to a decrease in gas volume (Figs. 9B and 9C) until the top-bottom percolation is interrupted (Fig. 9D). The pressure depletion mode starts with quick pressure drawdown to nucleate gas bubbles, which are kept small and non-percolating by immediately returning to a pressure near the bubble point (Fig. 9E).
Pressure decrease then led to growth of the gas bubbles (Figs. 9F and 9G) until top-bottom percolation was reached (Fig. 9H). The associated pressure-volume curves show a very similar signature as in [5].

\subsection{Flow experiments: injection of gas near bubble point}

Gas bubble expansion due to pressure depletion is only one limiting case of several possible flow regimes. Expansion of gas bubbles in one region/set of pores can cause flow and displacement in other regions/pores. In order to address this flow regime, a series of experiments was conducted where pure propane gas was injected into the propane-decane mixture at a pressure near the bubble point before any 


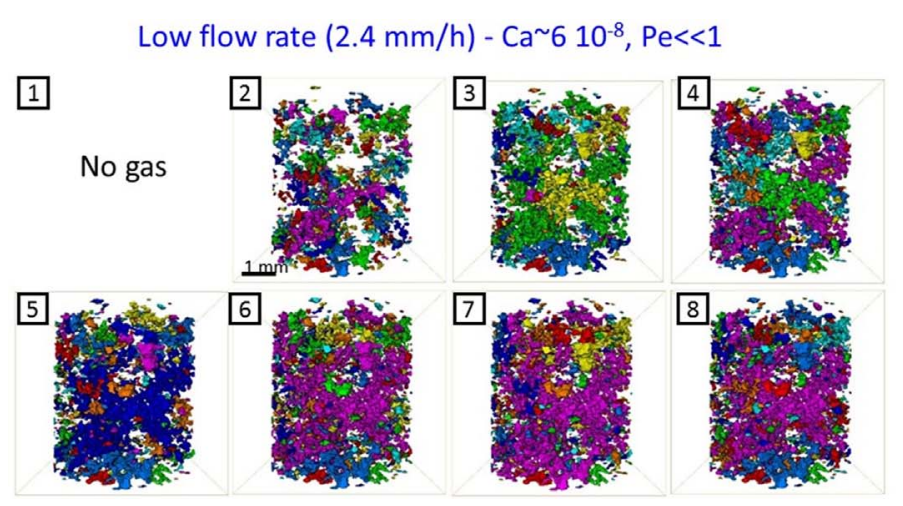

High flow rate $(72 \mathrm{~mm} / \mathrm{h})-\mathrm{Ca} 1.810^{-6}, \mathrm{Pe} 1$

Fig. 10. Micro-CT images (colors indicate individual gas clusters) for gas injection near the bubble point at low flow rates (left) and high flow rates (right). For the low flow rate experiments, the Peclet number $\mathrm{Pe} \ll 1$, while for the high flow rate experiments Pe $\sim 1$, which indicates that at low flow rates the mass transport of gas is dominated at diffusion while for high flow rates the front propagation follows the injection velocity.

gas bubbles were nucleated. Two classes of experiments were conducted where one was at a low flow rate and one at a high flow rate. Respective $3 \mathrm{D}$ gas clusters are displayed in Figure 10.

Starting point was the experiment at low flow rates where propane was injected into the $\sim 50: 50$ propanedecane mixture at a rate of $0.03 \mathrm{~mL} / \mathrm{h}$ or a Darcy velocity of $v=2.39 \mathrm{~mm} / \mathrm{h}$. The respective capillary number of $\mathrm{Ca}=\mu \cdot v / \sigma=6 \times 10^{-8}$ (for a liquid viscosity of $\mu=$ $0.296 \mathrm{mPa} s$ and an interfacial tension of $\sigma=17 \mathrm{mN} / \mathrm{m}$ ) which is typical for conditions in the reservoir far away from the wellbore. The distribution of gas shown in Figure 10 on the left shows initially no gas present, but already in the first step disconnected gas present everywhere in the pore space without any visible top-bottom connectivity. Top-bottom connectivity is only observed from scan 6 onwards. Note that persistent connectivity is not a necessary requirement for flow as there are displacement processes such as snap-off [41-43] and cooperative dynamics such as ganglion dynamics [44] that can lead to saturation distributions without persistent connectivity. However, such processes are extremely fast and respective time scales of a few milliseconds are not resolved in the $1 \mathrm{~h}$ time intervals accessible here. But there are some striking differences to examples from the literature on immiscible displacement where in drainage typically at least one percolating path is established while in the low-flow rate injection cases here no such percolating pathway is present for 5 subsequent scans (lasting over $5 \mathrm{~h}$ ) while significant saturation changes occur. That is beyond of what has been observed in immiscible drainage experiments where only occasionally a connection through a single pore throat was missing [42]. That raises the question whether other transport mechanisms are at play here. The main difference to 2-phase immiscible water-oil cases is that in the situation here there is immiscibility of 2-phase but mutual solubility and components partitioning between two phases. That allows e.g. the propane component to partition from the gas phase into the liquid and then become subject to diffusion as transport mechanism. One of the fundamental questions is whether for instance propane propagates faster by diffusion than by convection. A key figure is the so-called Péclet number,

$$
\mathrm{Pe}=\frac{L \cdot v}{D}
$$

which is the ratio of advective over diffusive flux, for a characteristic length scale $L$, advective velocity $v$, and diffusion coefficient $D$. For the low velocity injection case with an interstitial flow velocity of $v \approx 3.5 \mu \mathrm{m} / \mathrm{s}$ a characteristic length scale of $L \sim 60 \mu \mathrm{m}$ (typical pore body diameter for Bentheimer sandstone), a typical molecular self-diffusion coefficient for hydrocarbon mixtures of $D \approx 5.8 \times 10^{-9} \mathrm{~m}^{2} / \mathrm{s}[45]$ we obtain $\mathrm{Pe}=4 \times 10^{-2} \ll 1$ meaning that diffusive transport dominates over advection. That means that the propane injected as a gas can partition into the liquid mixture present in the pore space and advance by diffusion significantly faster than by the flow of the injected gas phase. In that way it can distribute across the whole field of view and increases the local propane concentration to a composition which potentially exceeds locally bubble point conditions. That would then lead to a phase split after which the gas phase as non-wetting phase fills big pores (except for a thin liquid film coating the surface of the grains, which can be seen as a thin rim in Figures $11 \mathrm{C}-11 \mathrm{~F}$ which is right at the resolution limit of the micro-CT scanner and not fully resolved) while the liquid phase occupies the pore throats - a situation as we observe it in our experiments. Note that for the Péclet number estimates the self-diffusion coefficient has been used to estimate the diffusive flux. Diffusion in concentration gradients can be substantially higher which would increase the diffusive flux, i.e. emphasize this mechanism even more.

In order to test this hypothesis, we conducted a second set of experiments where the injection rate is increased by a factor of $30(0.9 \mathrm{~mL} / \mathrm{h}$ injection rate or $72 \mathrm{~mm} / \mathrm{h}$ linear flow velocity) such that $\mathrm{Pe} \sim 1$ meaning that the diffusive transport is now slower than the advective transport i.e. the propagation of the immiscible displacement front. 


\section{Single-component system}

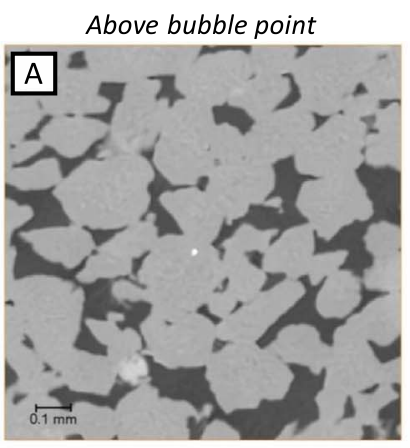

After gas nucleation

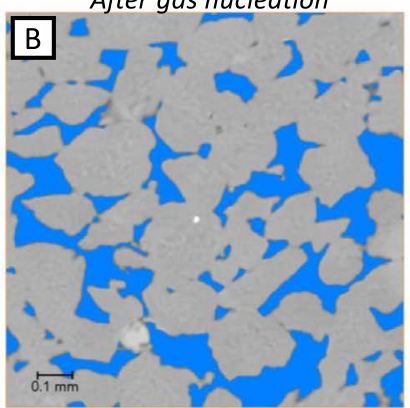

Two-component system
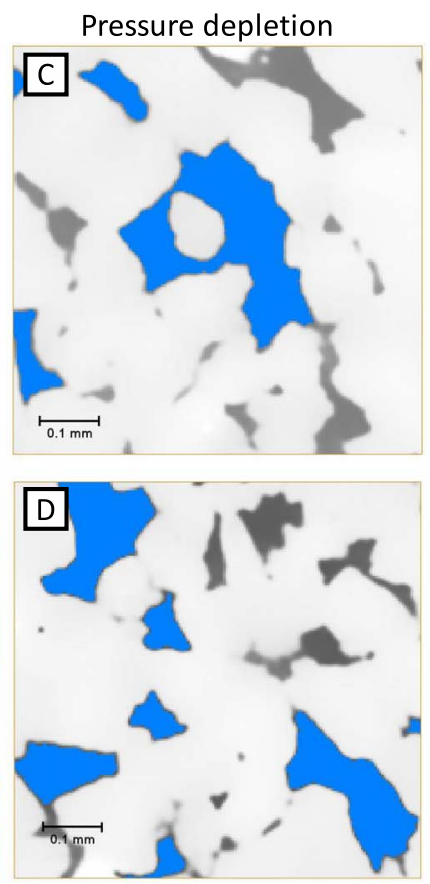

Fig. 11. Pore level fluid occupancy for a single component system (A, B) and a two-component system (C-F). In the singlecomponent case, above the bubble point the whole pore space is filled with liquid while below the bubble point pressure (A), after equilibration, the whole pore space in the field of view is filled with gas (B). In the two-component system, that behavior is distinctly different. In pressure-depletion experiments, below the bubble point pressure pores are either filled with liquid or with gas (C-F). There are no partially occupied pores like in normal water-oil displacement experiments (see e.g. Fig. 4 in [44] or Fig. 3 in [42]).
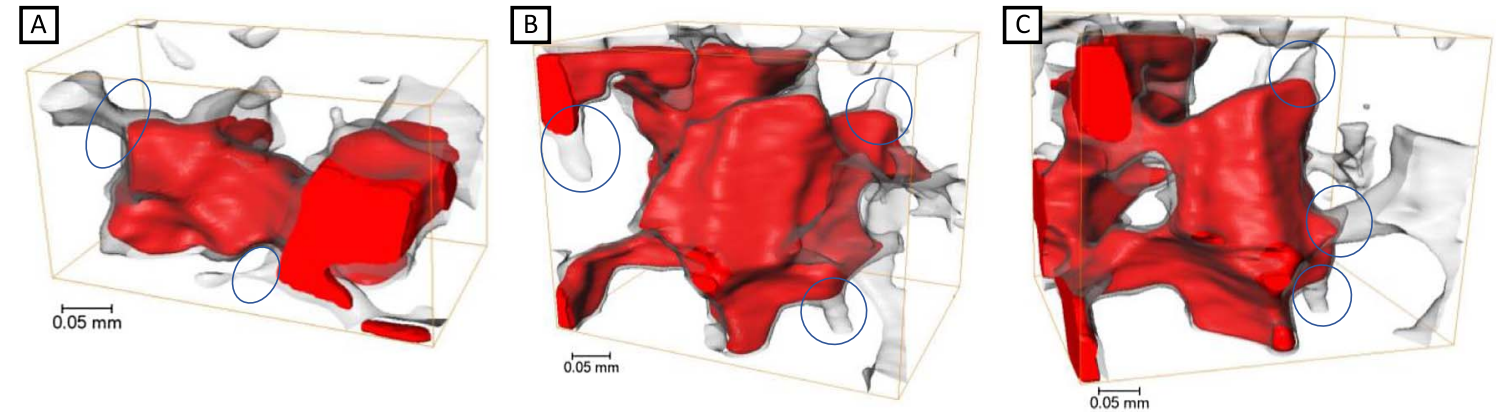

Fig. 12. 3D visualization of three representative gas clusters from pressure depletion experiments illustrating that pores are filled up to the respective pore throats by gas.

The respective 3D gas distribution is displayed in Figure 10 on the right-hand side where we observe an overall connected situation.

\subsection{Pore scale fluid configuration in the 2-component system}

In terms of pore level fluid occupancy there are distinct differences between the single and the two-component system. As shown in Figure 11, in the single-component case, above the bubble point the whole pore space is filled with liquid while below the bubble point pressure, after equilibration, the whole pore space in the field of view is filled with gas (see also Fig. 6). In the two-component system, that behavior is distinctly different. In pressure-depletion experiments (quick depletion-re-pressurization followed by depletion), below the bubble point pressure pores are either filled with liquid or with gas as shown in Figures 11A and 11B (not accounting for possible liquid wetting films which are typically below the resolution of micro-CT).

There are no or only relatively few partially occupied pores like in normal water-oil displacement experiments 
as displayed e.g. in Figure 4 in [44] or Figure 3 in [42]. Pores are either filled with liquid or gas up to the pore throat which can be seen in the 3D rendering of three representative gas clusters shown in Figure 12.

The final state is reminiscent of gas bubbles growing always until the capillary resistance of the pore throats is reached. In an unrelated experiment with much higher temporal resolution (10 s intervals for full 3D scan, obtained at a synchrotron beamline) the evolution of an presumably air bubble inside brine shows similar steps (see Fig. 4 in [46] also together with previous studies illustrating that bubbles are subject to homogeneous nucleation and not on rough surfaces by e.g. a surface reaction [47]) where the menisci of the growing bubble are held up at pore throats. This illustrates that pore throats represent natural barriers in the growth of gas bubbles.

However, that does not fully explain the distribution of largely disconnected gas bubbles in either entirely filled (except for a thin liquid wetting films) or completely empty pores observed in the 2-component system in Figures 11C$11 \mathrm{~F}$. If every pore eventually develops a nucleation seed one would expect that pores are more evenly occupied with occupancy according to the phase fractions defined by the equation of state for the conditions, which here is a 50:50. But that is not what is observed. The pore occupancy statistics of gas bubbles (which was obtained by mapping the pore scale images of e.g. Figures 9 and 10 onto a pore network [48]) shown in Figure 13 shows a selective occupancy of gas bubbles in intermediate size pores while smaller pores are not filled at all, which is not what one would expect from a homogeneous nucleation mechanism.

There are also distinct differences between the pressure depletion and the gas injection experiments. In the low rate gas injection experiment we see over time a growth of the distribution in terms of numbers of pores occupied but also the width of the distribution mainly into smaller pores. In the pressure depletion experiment we see that the number of pores in the size range of $45<D<60 \mu \mathrm{m}$ increases, but the occupancy of smaller pores decreases.

The experimental observation is more reminiscent of the ripening and likely also anti-ripening processes described in the groundbreaking work of [49]. Ripening is an interplay of diffusive transport and (capillary-) pressure based partitioning equilibrium between different fluid phases [50]. In the bulk i.e. outside a porous medium, Ostwald ripening would imply that large gas bubbles grow on the cost of smaller ones. From a thermodynamics perspective, Ostwald ripening is a process where diffusive transport from one gas bubble to another gas bubble through an in-between liquid (in which the gas is soluble) occurs towards equilibrium, i.e. equality of chemical potential in each phase component and equality of bubble capillary pressures. This has different implications in a porous medium, where in addition to the solid fraction constraining the fluid phases to the pore space, the pore body-pore throat geometry impacts the possibility for e.g. gas bubbles to grow by ripening beyond the size of individual pores [49]. Even though diffusion is only impacted in the percent range by the geometric constraint of the pore space, the increase in curvature and the associated (capillary) pressure for growth past a pore

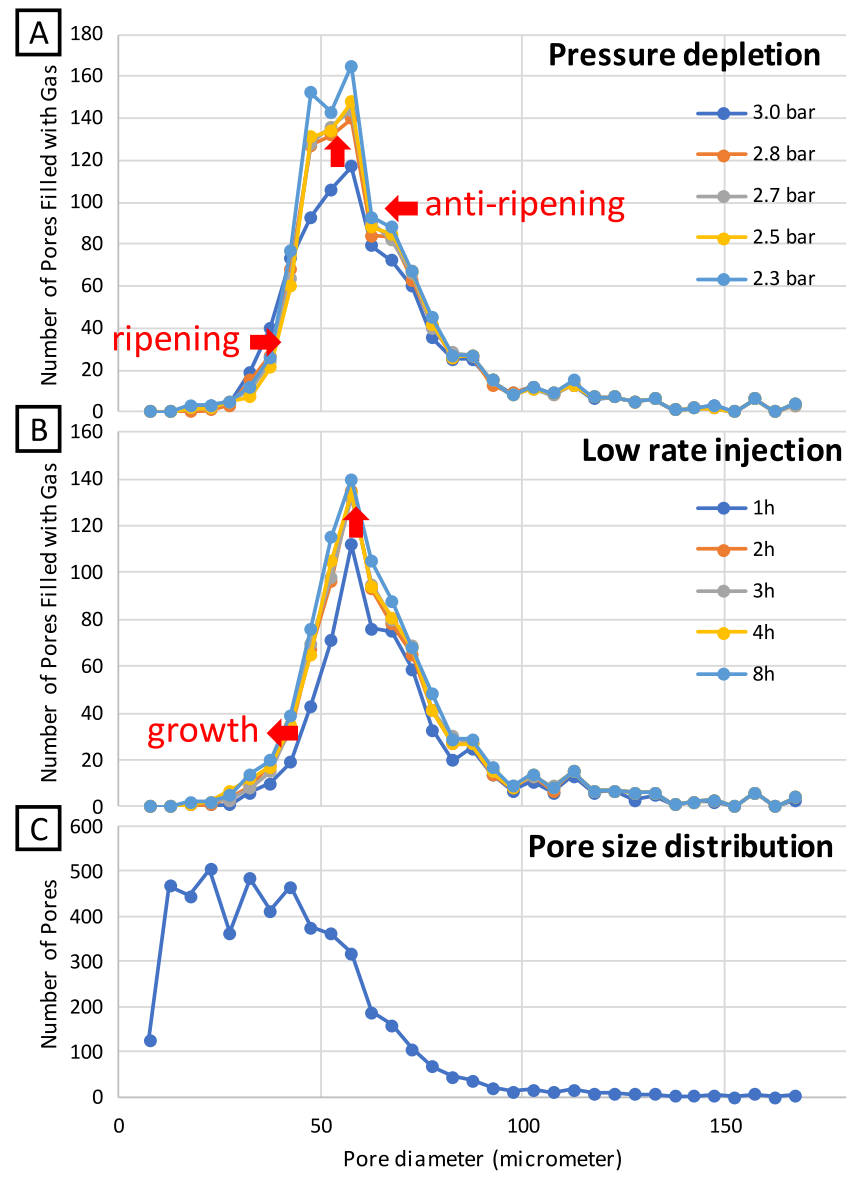

Fig. 13. Pore level occupancy distribution of gas filled pores in pressure depletion (A) and gas injection experiments (B). In both cases a preference for intermediate-sized pores is observed in comparison with the pore size distribution (C). In the low rate injection experiment we see over time a growth of the gas bubble size distribution in terms number of pores occupied and the width of the distribution (B). In the pressure depletion experiment we see over time a selective increase of gas bubble occupancy in intermediate-size pores while the occupancy in small pores decreases (A). This is a signature of ripening effects. The fact that the size distribution does not (or only very marginally) grow into larger pores suggests also the presence of anti-ripening effects [49].

throat causes a de-facto anti-ripening effect with pore throats acting as barriers for growth [49] which can only be overcome by a significant driving forces, e.g. significant gravity potential over large column height [51]. Ultimately, both phenomena of ripening and anti-ripening are the result of the same underlying physical process of coarsening in porous media.

The differences between the pressure depletion experiments and low rate injection experiments in terms of pore occupancy statistics observed in Figure 13 may be explained by the presence of convective transport in the injection experiment which interferes with ripening and anti-ripening processes in terms of the component concentration in the liquid phase. In the pressure depletion 

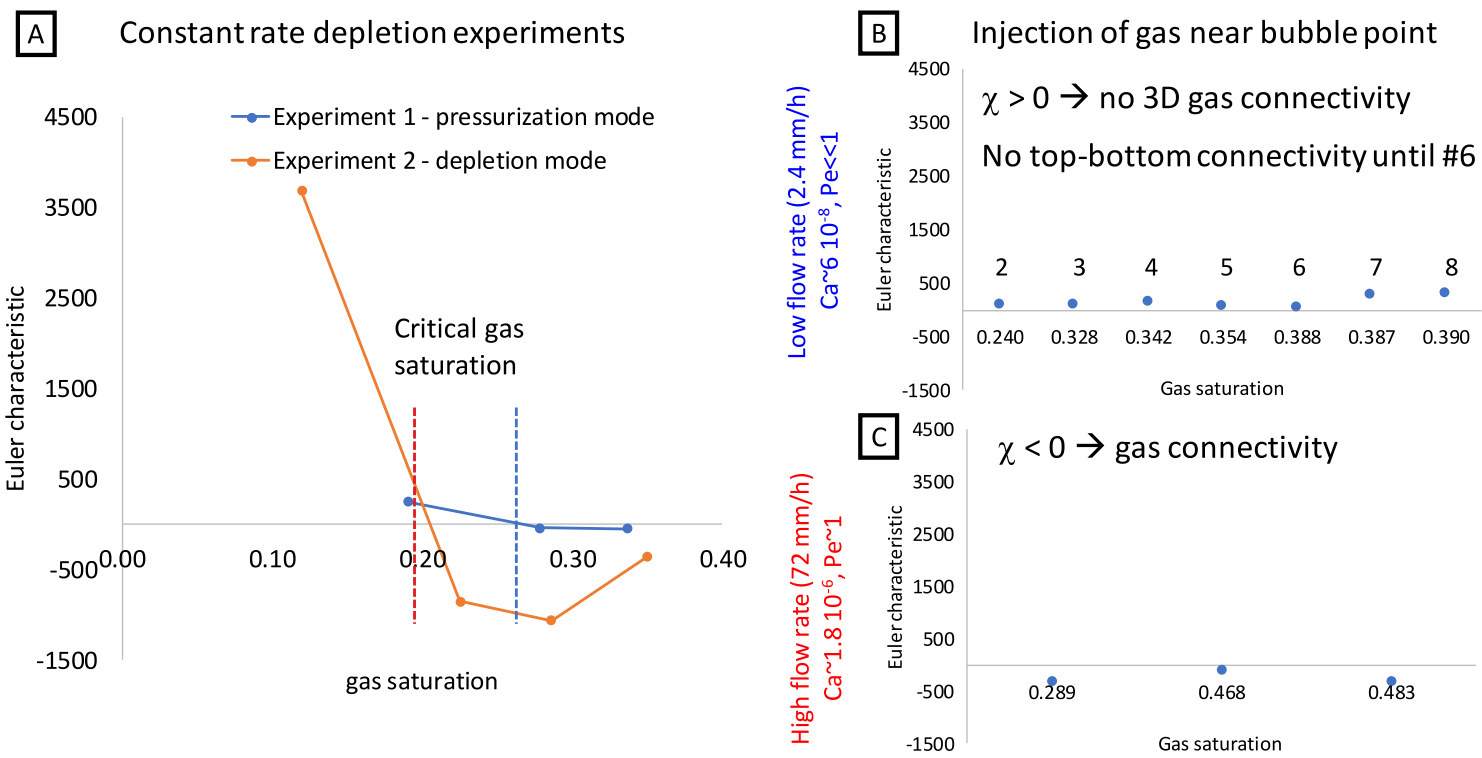

Fig. 14. Euler characteristic of the gas clusters - which is a measure for connectivity - vs. gas saturation for the pressure depletion experiments (left) and gas injection experiments (right). Euler characteristic $\chi>0$ indicates disconnected gas clusters while $\chi<0$ indicates connectivity. $\chi=0$ coincides approximately with the percolation threshold, and hence serves as an indication for the critical gas saturation $S_{g c}$. For the pressure depletion experiments (A) the critical gas saturation $S_{g c}=0.2$ for pressure depletion and $S_{g c}=0.25$ for re-pressurization, which is overall in the range of percolation thresholds for porous objects in 3D. For the gas-injection experiments we observe an overall not-connected $(\chi>0)$ situation for the low injection case $(B)$ and an overall connected situation $(\chi<0)$ for the high injection case $(\mathrm{C})$.

experiment such convective transport is not present at all, i.e. in absence of external convection where the transport is entirely controlled by diffusion which may make ripening effects more dominant.

\subsection{Connectivity analysis}

A key aspect of the interpretation of the 3D fluid configuration is the analysis of the connectivity. While top-bottom connectivity can be directly identified from the cluster analysis (same color cluster connected to both top and bottom), for the reservoir a more isotropic connectivity may be more relevant. A convenient and easy-to-compute quantity is the Euler characteristic,

$$
\chi=\text { objects }- \text { loops, }
$$

which is a measure for connectivity and also a state variable for multiphase flow in porous media [52-54]. In this study $\chi$ is computed for the gas phase from the micro-CT images using Avizo [52]. $\chi=0$ marks the percolation threshold which in the case of the pressure depletion experiments is an indication for the critical gas saturation $S_{g, c}$. In Figure $14 \mathrm{~A}$ we plot $\chi$ as a function of gas saturation $S_{g}$ and obtain zero crossings i.e. critical gas saturation of $S_{g c}=0.25$ for the pressurization mode and $S_{g c}=0.2$ for the pressure depletion mode. The fact that the critical gas saturation in pressurization and depletion are not identical suggests that re-pressurization is not simply the reverse process of depletion, which is ultimately caused by the same asymmetry of pore scale displacement processes that cause hysteresis in capillary pressure [53-55] and in addition the hysteresis caused by super-saturation [12].

That range of critical gas saturations from the pressure depletion/re-pressurization experiments is consistent with the range of typical percolation thresholds of $3 \mathrm{D}$ structures as listed in Table 1. However, the associated saturations are significantly higher than the onset of gas mobility observed in experiments that involve flow [2]. That indicates that the problem of critical gas saturation may involve other processes, as already suggested in the discussion around the low injection rate experiment from Figure 10 left.

When applying the same connectivity analysis to the injection experiments from Figure 10 for the low rate gas injection experiment overall positive values for the Euler characteristic $\chi>0$ are obtained indicating no or a lesser degree of connectivity (Fig. 14B) while the high rate injection has negative values for the Euler characteristic $\chi<0$ indicating a connected path (Fig. 14C).

The main conclusion from the connectivity analysis is that the two injection experiments differ conceptually in connectivity. Increasing the flow rate by a factor of 30 does not change the visco-capillary balance (the capillary numbers $\mathrm{Ca}=6 \times 10^{-8}$ and $\mathrm{Ca}=1.8 \times 10^{-6}$ are both still in the capillary dominated regime). Also, the fluid viscosity ratios are the same. Therefore, according to Lenormand's stability diagram [24] the root cause for an onset of gas mobility below the percolation threshold is less likely related to a change in capillary-viscous flow regimes such as viscous fingering [24]. But what does change is the ratio of advective over diffusive transport, expressed by the Péclet number (from $\mathrm{Pe} \ll 1$ to $\mathrm{Pe} \sim 1$ ). The $\mathrm{Pe} \sim 1$ case 
Table 1. Percolation thresholds of porous structures and lattices in 3D.

\begin{tabular}{lccr}
\hline Structure & Coordination number & Percolation threshold & Reference \\
\hline Simple cubic lattice & 6 & $0.30-0.31$ & {$[15-18]$} \\
BCC lattice & 8 & $0.243-0.246$ & {$[15-18]$} \\
FCC lattice & 12 & $0.195-0.199$ & {$[19]$} \\
Sphere pack & 6 & 0.31 & {$[20]$} \\
\hline
\end{tabular}

indicative of a more advection dominated regime has indeed the higher connectivity, while the $\mathrm{Pe} \ll 1$ case has $\chi>0$ suggesting transport without permanent connectivity of the gas phase, which is possible by diffusive transport of the gas dissolved in the liquid phase.

\subsection{Relative permeability}

Besides the critical gas saturation $S_{g c}$, the associated liquidgas relative permeabilities are perhaps even more important parameters, in particular for dynamic modelling $[8,9,56]$. Because of all the complications already discussed, experimental measurements of relative permeability by applying pressure gradients causing flow are problematic, as this starts interfering with pore scale flow regimes. In order to avoid this additional uncertainty, the experimental approach used here allows to compute the (connected pathway) relative permeability from the $3 \mathrm{D}$ spatial distribution of liquid and gas phases obtained from segmented micro-CT images. By using the MRT-LBM method [37] described in Section 2.4, the absolute permeability of the total pore space and the permeabilities of liquid and gas phases are computed following a methodology similar to that in [57]. The relative permeability of gas is then the result of the permeability computation for the gas connected pathway from the micro-CT image, divided by the absolute permeability computed from the whole pore space. The relative permeability of the liquid is computed in an analogous way, using instead the segmented liquid phase from the micro-CT scans. For gas-liquid scenario where gas is a completely non-wetting phase, the connected pathway contribution to relative permeability dominates over the flux contribution of ganglion dynamics which can be therefore neglected [30, 31].

The results are shown in Figure 15 on a linear (top panel) and logarithmic scale (bottom panel). The left column shows the results from the pressure depletion and re-pressurization experiments. The middle column shows the same data but for comparison with water-oil drainage and relative permeability curves for a water-wet $\left(1.5^{\prime \prime}\right.$ diameter) twin sample of the same rock superimposed. Note that the saturation scale in Figure 15 is the liquid saturation, which is 1-total gas saturation (connected and disconnected). Therefore, we can observe situations where $k_{r, g}=0$ for $S_{o}<1$.

The gas-liquid relative permeabilities are compatible with a typical Corey behaviour [26] with exponents $n_{w}>1$ and $n_{o}>1$, i.e. not straight lines it would be in near-miscible situations [56], this makes sense because in the propane-decane system at our experimental conditions, i.e. far away from the critical point, the liquid-gas interfacial tension at the bubble point is about $17 \mathrm{mN} / \mathrm{m}$, meaning that there are significant capillary forces. The water-oil drainage $k_{r}$ is shown as solid red and blue lines, the imbibition $k_{r}$ is shown as dashed lines. In the critical gas experiment, the liquid phase is the wetting phase while gas is the non-wetting phase (gas is always the least wetting phase). Therefore critical-gas liquid relative permeability should be comparable with the water relative permeability, and gas with the oil relative permeability. The gas relative permeability follows a drainage relative permeability curve when re-scaling the saturation endpoint to the critical gas saturation $S_{q \text { crit }}=0.21$ obtained from the $\chi=0$ transition in Figure 14A (and a Corey exponent of $n_{o}=1.8$ ). The main reason is that a critical gas saturation is required before gas becomes mobile, i.e. a relative permeability can be defined. Therefore, the mobile gas saturation range starts at critical gas saturation which is larger than zero (hence the gas relative permeability cannot be compatible with a primary drainage curve) and smaller than a typical residual oil saturation (hence not compatible with a first imbibition relative permeability).

The liquid relative permeability follows more wettingphase imbibition curve (which is an extension of the water imbibition relative permeability using a Corey representation with an exponent $n_{w}=4.5$ ) but is overall also compatible with a drainage curve. The relative permeability for gas injection (left column) clearly deviates from the pressure depletion curves, indicating that gas injection follows a different transport mechanism. There is hardly any hysteresis visible between pressurization and pressure depletion mode. Even though the pressurization model corresponds to an imbibition and the depletion to a drainage process, the saturation range is probably too small to show significant hysteresis. The dynamic range was also too small to establish a $k_{r, g}(\chi)$ relationship as in [58].

The relative permeability for the gas injection experiment are superimposed in the right column of Figure 15. The liquid relative permeability does follow a similar trend as for the pressurization/depletion experiments, but the gas relative permeability is clearly different and there is also no obvious re-scaling with percolation-based critical gas saturation values. That could indicate that indeed there is a different transport mechanism at play.

\section{Discussion}

A key advantage of the experimental approach with pore scale in-situ imaging is that the gas distribution and 

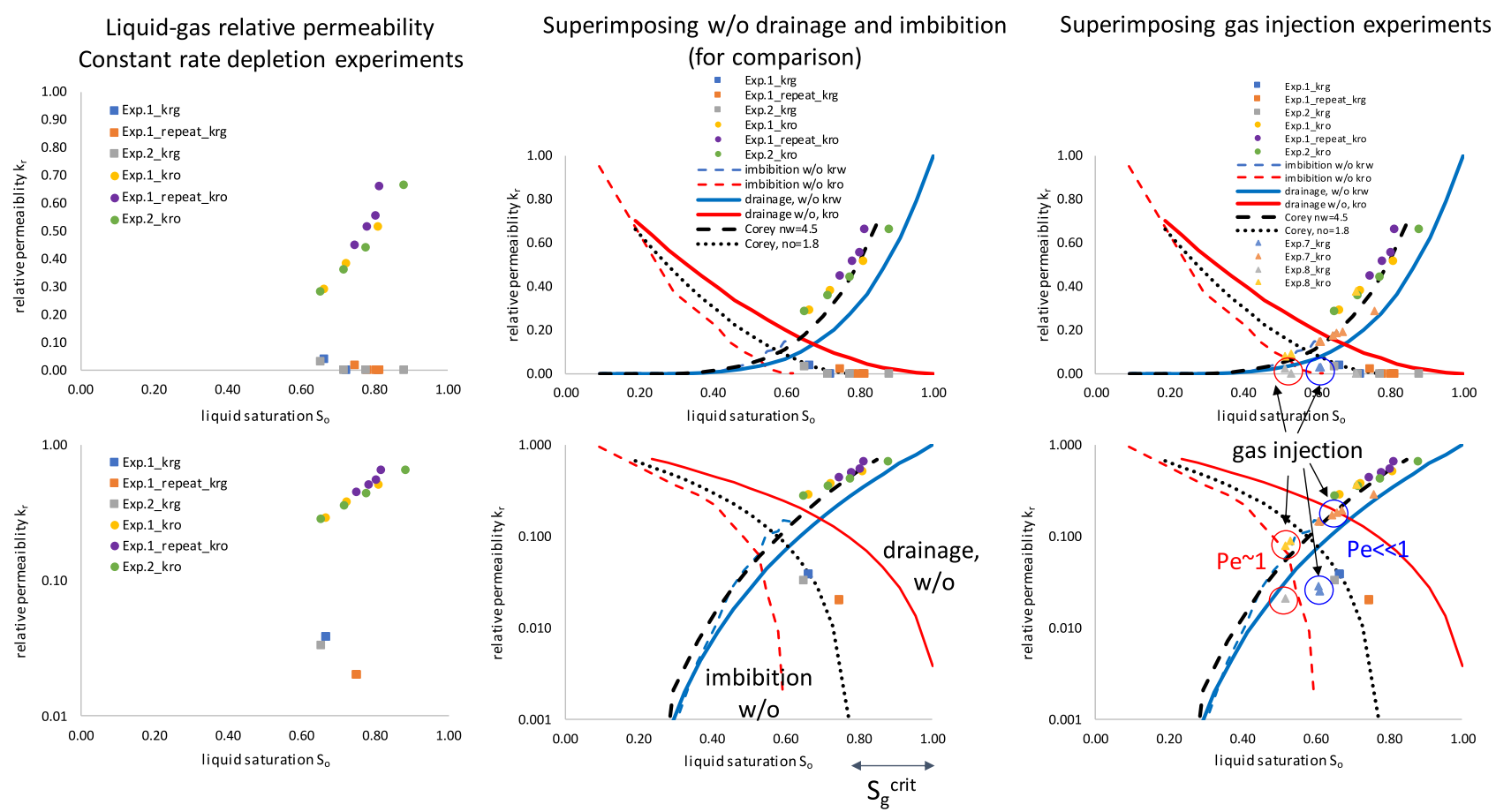

Fig. 15. Relative permeability for liquid and gas (left column) computed by single-phase flow simulations (LBM method) on the fluid distribution obtained from micro-CT. The gas relative permeability follows a drainage relative permeability curve when re-scaling the saturation endpoint to the critical gas saturation (middle column). The liquid relative permeability follows more wetting-phase imbibition curve but is overall also compatible with a drainage curve. The relative permeability for gas injection (left column) clearly deviates from the pressure depletion curves, indicating that gas injection follows a different transport mechanism.

connectivity can be directly determined and in this way effects of pressure depletion and flow on gas mobility can be separated. For the connected pathway contribution relative permeability can be obtained without the need of applying pressure gradients which perturbs the phase equilibrium. At the same time, it allows gaining more detailed insight into the complexity of the critical gas saturation problem. We can clearly observe that the depression of the bubble point observation is directly linked to the absence of nucleation seeds and after these have been created by a rapid pressure drawdown the so-nucleated gas bubbles expand more continuously as expected from equilibrium phase behavior.

One of the key learnings is perhaps that constant rate pressure depletion leads, irrespective of depletion rates, to critical gas saturations compatible with 3D percolation thresholds such as in random sphere packs i.e. in the range of $20-30 \%$, which is not consistent with observations under conditions that involve flow, i.e. both on the experimental and the field scale. That raises the question whether such constant rate depletion experiments cover the relevant flow regime.

The nucleation of gas bubbles is followed by gas expansion which is accompanied by a volume increase of the gas phase that causes flow and displacement processes in the adjacent pore space. In order to address this flow regime, also the injection of propane gas into the gas-propane liquid near the bubble point (in absence of initial gas bubbles) was investigated. Injecting gas at a high rate (representative to somewhat closer to the producing well) leads to a more traditional immiscible (drainage) displacement process where the gas phase is overall more connected (Euler characteristic $\chi<1$ ). However, injection at a 30 times lower flow rate which is somewhat more representative to a location deep in the reservoir leads to a 3D gas distribution without persistent connectivity throughout the rock, i.e. a saturation of gas mobility below the percolation threshold (Euler characteristic $\chi>1$ ). While the low and the high injection rate experiments fall both within the same flow regime in terms of capillary number, they differ in terms of Péclet number $\mathrm{Pe}$. For the low injection rate $\mathrm{Pe} \ll 1$ meaning a much more diffusion-dominated regime, where the gas injected above the bulk liquid bubble point pressure can dissolve into the liquid phase at the injection front and change the respective composition such that the bubble point pressure increases. This mechanism could be an explanation of why the presence of flow gas mobility is observed already below the percolation threshold. This reasoning, based on the experimental evidence, is sketched in a potential mechanism in Figure 16.

The pore level fluid occupancy was observed to differ systematically from 2-phase immiscible displacement experiments where typically a wider range of pore level fluid occupancy is observed ranging from pores filled fully with wetting or non-wetting fluid (except for films of the wetting fluid $[59,60]$ which is a consequence of the liquid phase being in our experiments always the wetting phase, see dark rim around the grains in Figures $11 \mathrm{C}-11 \mathrm{~F}$ representing the 


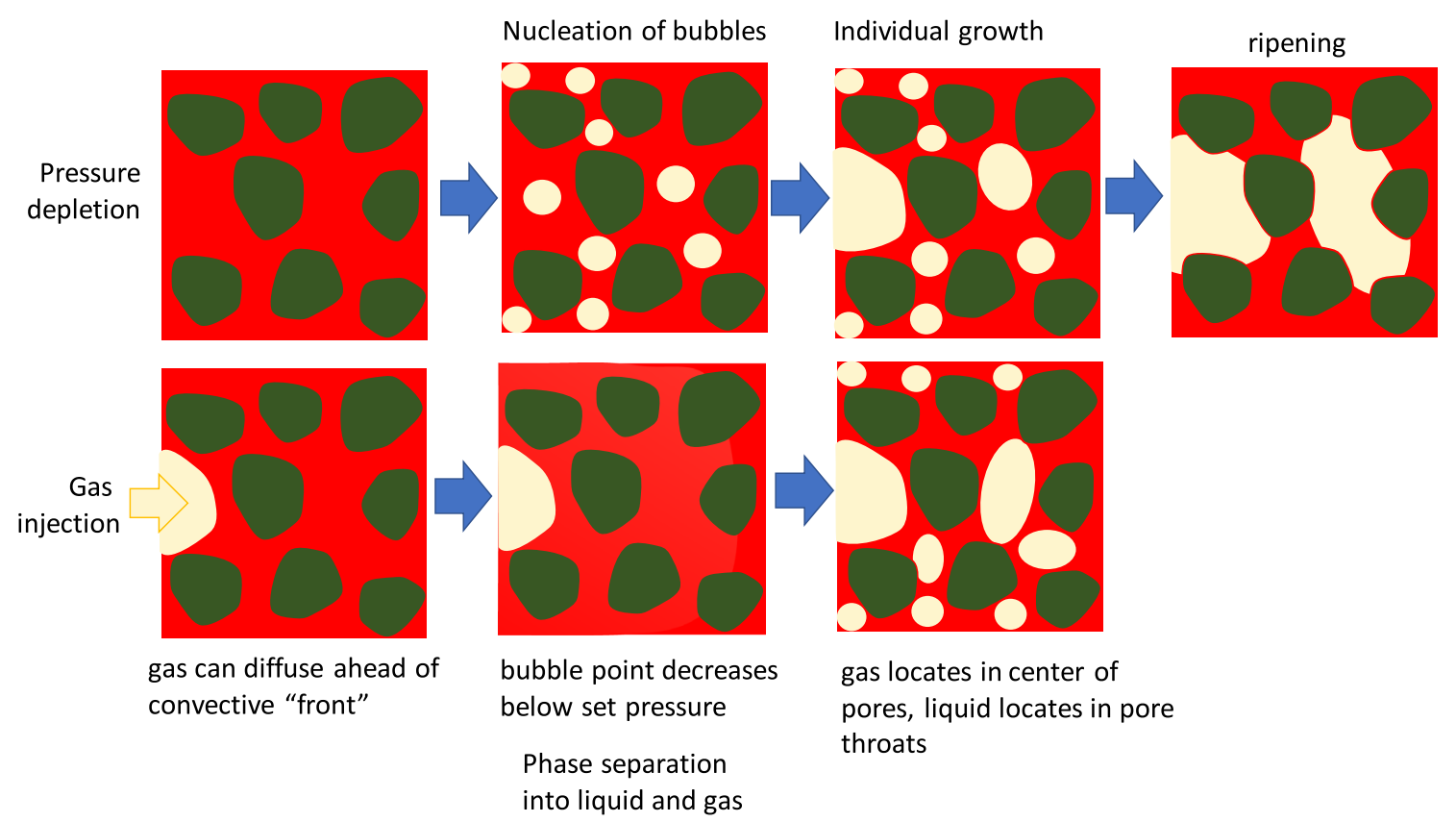

Fig. 16. Illustrations of the evolution of the pore scale fluid distribution. During pressure depletion experiments, at some point gas bubbles are nucleated which initially may grow, but ripening will on the long run cause smaller bubbles to disappear and larger bubbles grow until individual pores are completely filled. Both for grow and ripening, pore throats act as natural barriers [49]. Upon injection of gas at or near the bubble point, gas diffuses ahead of the "convective front". That changes the local composition such that the bubble point decreases below the local conditions. That leads to a phase separation into liquid and gas. The gas then locates in the center of pores while the liquid (that preferentially wets the solid) locates in the pore throats. In this way gas advances, i.e. is mobile, without having a permanent connectivity. That distribution will then undergo similar ripening processes as in the pressure depletion case.

liquid phase) but also partially filled pores are observed $[42,44]$. Instead, we see only pores filled completely with either gas (except for the liquid wetting film on the solid grain surface) or liquid, but no partial occupancy is observed. That is on the one hand caused by pore throats being a natural barrier for the expansion of gas bubbles [49]. But one would still expect a more even distribution of pore level occupancy with gas/liquid fraction defined by the equation of state (which at experimental conditions would be around 50:50). The observed distribution of either fully gas or liquid filled pores is more reminiscent of the final state of a ripening/anti-ripening process in porous media where pore throats act as thermodynamic barriers for the Ostwald-ripening caused growth [49]. This is also visualized in Figure 16.

That means that the generally accepted view that at critical gas saturation a continuous, sample spanning, gas phase is established is put to question. Mobility of gas is possible without connectivity well below the percolation threshold because of a range of mechanisms. These range from pore-scale capillary instabilities where gas clusters move as a discontinuous phase $[28,29]$ by ganglion dynamics $[30,31]$ to a more complex interplay between advective and diffusive transport with phase behavior and (anti-) ripening effects.

The open question is still what will happen at the reservoir level with associated length and time scales of e.g. pressure decline and whether the simple extrapolation of the rate dependency accessible in the laboratory to field conditions as shown in Figure 2A is really valid. The extrapolation is based on the picture of gas bubble growing to a connected phase and the competition between pressure decline rate and the kinetics of gas bubble nucleation and growth. From the experimental observation we can already rule out that the kinetics of growth is the limiting effect because it occurs almost instantaneously, i.e. at a time scale well below $1 \mathrm{~h}$ (which compared with the pressure decline rate at the field scale is practically instantaneous). The bubble nucleation might still be the rate limiting step defining the characteristic time scale, or in other words the question is whether in the laboratory experiments the gas bubbles would eventually have formed at the bulk phase thermodynamic bubble point if we had waited long enough.

There are several arguments that speak against that. One is that in homogeneous nucleation a nucleation seed can only grow from a critical radius on because the thermodynamic driving force $\Delta G_{V}$ defined by the over-heating/pressure below bubble point from the equation of state needs to overcome the energy penalty of the interface. The driving force scales with volume i.e. $\Delta G_{V}=-\Delta g_{V} \cdot \Delta V \propto-r^{3}$ where $r$ is the radius of the nucleation seed and $\Delta g_{V}$ is the difference in free energy per volume between the liquid and the gas state. The interfacial energy scales with $\Delta G_{S}=\sigma \Delta A \propto r^{2}$ where $\sigma$ is the 

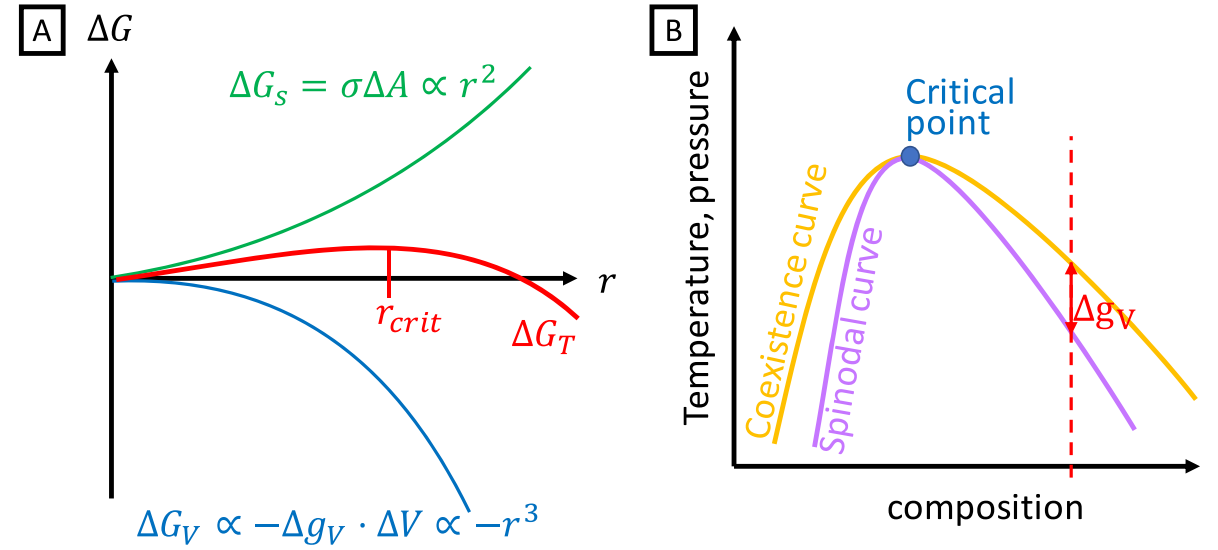

Fig. 17. Illustrations of the thermodynamics of bubble nucleation where a fluctuation can grow from a critical radius $r_{\text {crit }}$ onwards when the thermodynamic driving force for the phase separation $\Delta G_{V}$ overcomes the energy penalty for forming an interface [61, 62] (A). As a consequence, the phase equilibrium defined by the respective equation of state represents only the equilibrium co-existence of pre-existing phases, while phase separation occurs when a single phase below that coexistence condition becomes unstable against nucleation or fluctuations by e.g. spinodal decomposition [66] (B).

interfacial tension. $\Delta V$ and $\Delta A$ are the volume and area of the nucleation seed, respective. The resulting free energy $\Delta G_{T}=\Delta G_{V}+\Delta G_{S}=-\Delta g_{V} \cdot \Delta V+\sigma \Delta A$ allowing growth of the nucleation seed from a critical radius $r_{\text {crit }}$ onwards [61-63] as illustrated in Figure 17A.

Fluctuations larger than $r_{\text {crit }}$ typically don't form spontaneously at the bulk phase thermodynamic bubble point as defined per equation of state. This condition represents strictly speaking the condition where pre-existing phases can co-exist, but does not describe the condition where phase separation would occur spontaneously [64]. Only when nucleation is facilitated as often done in experimental PVT setups, phase separation at the bulk bubble point condition is observed. However, if no special measures are taken, fluids can significantly over-heat resulting in boiling point retardation accompanied by a sudden volume expansion of the formed vapor phase as consequence of an instability [64]. Unless special measures taken to prevent that (e.g. adding stones with micro pores, the same effect occurs in champagne glasses where $\mathrm{CO}_{2}$ bubbles are nucleated in hollow cellulose fibers originating from the cloth used to dry the glasses after washing [65]) the gas nucleation is an unstable process where phase separation only occurs at a condition away from the equilibrium co-existence line. This is illustrated in Figure 17B using the example of spinodal de-composition as mechanism for phase separation $[66,67]$. For bubble nucleation a somewhat similar logic is valid because the critical nucleation seed radius $r_{\text {crit }}$ can be reduced by increasing the driving forces $\Delta g_{V}$ which is proportional to the over-heating or pressure depletion below the bubble point pressure i.e. $\Delta g_{V} \propto\left|p-p_{\text {bubble }}\right|$. Once one critical nucleation seed is formed, the phase separation occurs spontaneously and the phase split occurs into fractions defined by the thermodynamic conditions required for the formation of the critical nucleation seed, which are below the co-existence line. That means that a nucleation mechanism will always result in a significant phase split. In other words, except for the critical point, it is thermodynamically not possible to have gas coming out of solution in a sequence of equilibrium steps where first very small bubbles form. The formation of gas bubbles and their growth is a non-equilibrium process with very fast kinetics until the phase split at respective over-heating/pressure below bubble point required for successful nucleation is reached, i.e. represents an instability [64]. In our experiments in Figures 6-9 we observe exactly this behavior. For the single component case, the equilibrium condition is only reached when all liquid is converted into gas which is what we also see in terms of pore scale fluid occupancy in Figure 11A. For the 2-component system it is not possible to achieve gas nucleation condition at the thermodynamic bubble point. Only by actively nucleating small gas bubbles by fast pressure drawdown, we can achieve co-existence of gas bubbles at the thermodynamic bubble point.

That puts into question the whole picture about gas bubbles first nucleating and then growing slowly and successively until percolation is reached. In pressure-depletion experiments, nucleation happens almost instantaneously and to a significant extent where - depending on initial composition - percolation is instantaneously reached. That would imply that in many cases it is simply not possible to explain the mobility of gas well below the percolation threshold by a connected pathway argument. In addition, the explanation that in laboratories the pressure decline rate is always faster than the equilibrium growth of gas bubbles is not valid either. In other words, the gas nucleation and growth is not a kinetically-controlled process but governed by thermodynamic conditions as illustrated in Figure 17. The kinetics after successful nucleation is dominated by the ripening effects which occurs at a time scale of few hours [49]. That picture does not provide a motivation for extrapolations to lower and lower rates, i.e. longer than the time scale after which we see in the experiments bubbles having reached their stable configuration and ripening being largely complete. That raises 
questions about basis for the extrapolation to "field rates" for estimating critical gas saturation. It is possible that at field scale large column height can lead gravity-induced ripening [51] creating possible connected paths and associated mobility. The growth kinetics, however, is diffusion limited which is expected to occur on a time scale of years at relevant length scales [51].

To gain deeper insight, it might be helpful to extent current simulation capability to the level of pore-scale compositional simulation, i.e. multi-phase and multicomposition, with equation-of-state controlled phase behavior and partitioning of components between phases. Such simulation capability which is based on a density functional approach has been already demonstrated $[68,69]$. It would be very interesting to apply this approach to the problem of gas nucleation and gas mobility.

\section{Summary and conclusion}

This study provides fundamental insight into the gas dynamics in two-component systems that fall below the bubble point pressure and gas is nucleated. An experimental workflow is presented where the pore level gas and liquid distribution is imaged in 3D by X-ray computed microtomography while lowering the pressure below the bubble point or injecting gas. The pore level gas and liquid distribution gives direct access to the connectivity of the gas and hence its mobility. Respective (connected pathway) relative permeability is directly computed from the imaged gas and liquid distribution using lattice Boltzmann-based flow simulation. The gas relative permeability follows a classical non-wetting phase drainage relative permeability curve when re-scaling the saturation endpoint to the critical gas saturation. The liquid relative permeability is compatible with both a wetting phase imbibition or drainage relative permeability.

For pressure depletion in absence of flow critical gas saturations between 20 and $30 \%$ is observed which is consistent with typical percolation thresholds in 3D porous structures. However, that does not explain experimentally observed critical gas saturations significantly below $20 \%$ [2].

The pore level fluid occupancy is significantly different than in normal 2-phase immiscible systems as pore bodies are in almost all cases filled completely with either gas (except for wetting films which are below micro-CT resolution) or liquid phase while in 2-phase immiscible experiments we also see partial occupancy caused by displacement processes such as snap-off. This observation indicates that likely other mechanisms play a role in establishing gas mobility at saturations significantly below $20 \%$.

For gas injection experiments we observe evidence for gas mobility below typical percolation thresholds. The mechanism likely involves diffusive transport as respective Péclet numbers are significantly below one at field relevant flow rates. The consequence of diffusive transport are compositional gradients where locally the composition is such gas nucleation may occur. That would lead to gas mobility ahead of the convective front without connectivity of the gas phase being required. Furthermore, diffusive exchange leads to ripening and anti-ripening effects which explains to some extent also the pore level occupancy. The resulting relative permeability computed from the imaged fluid distributions using a lattice Boltzmann approach show distinctly different behavior between pressure depletion and flowing conditions.

These findings suggest that capillarity in a gas-liquid hydrocarbon mixture is far more complex than in a 2-phase immiscible system. Capillarity is coupled to phase behavior thermodynamics and kinetics on a fast time scale and diffusion-dominated mechanisms such as ripening and antiripening effects at a slow time scale. While the consequences for the current experimental and field modelling approaches are not yet fully clear, this shows that more research is needed to fully understand these effects and their implications.

Acknowledgments. We acknowledge Arjen Cense, Keith Love and Birol Dindoruk for helpful discussions that guided the experiments, and Willem-Bart Bartels and Hassan Mahani for designing and testing the micro-CT flow cell.

\section{References}

1 Sahni A., Gadelle F., Kumar M., Tomutsa L., Kovscek A. (2004) Experiments and analysis of heavy oil solution gas drive, SPE Reserv. Eval. Eng. 7, 03, 217-229. SPE-88442.

2 McPhee C., Reed J., Zubizarreta I. (2015) Core analysis: A best practice guide, Vol. 64 (1st ed.), Elsevier.

3 Scherpenisse W., Wit K., Zweers A.E., Snoei G., Van Wolfswinkel A. (1994) Predicting gas saturation build-up during depressurisation of a North Sea oil reservoir, in: Presented to the SPE Europec Meeting, London, Oct. 1994. SPE 28842.

4 Ligthelm D.J., Reijnen G.C.A.M., Wit K., Weisenborn A.J., Scherpenisse W. (1997) Critical gas saturation during depressurisation and its importance in the Brent Field, in: 1997 Offshore Europe Conference Held in Aberdeen, Scotland, 9-12 September 199\%. SPE-38475.

5 Firoozabadi A., Ottesen B., Mikklesen M. (1989) Measurements and modelling of supersaturation and critical gas saturation, in: SPE 19694, San Antonio.

6 Kamath J., Boyer R.E. (1995) Critical gas saturation and supersaturation in low-permeability rocks, in: SPE 26663, SPE Formation Evaluation, pp. 247-253.

7 Du C., Yortsos Y.C. (1999) A numerical study of the critical gas saturation in a porous medium, Trans. Porous Med. 35, $205-225$.

8 Egermann P., Vizika O. (2000) Critical gas saturation and relative permeability during depressurization in the far field and the near-wellbore region, in: 2000 SPE Annual Technical Conference and Exhibition held in Dallas, Texas, 1-October 2000. SPE-63149.

9 Egermann P., Vizika O. (2001) A new method to determine critical gas saturation and relative permeability during depressurization in the near-wellbore region, Petrophysics 42, 4, 352-361.

10 Piccavet N., Long J., Hamon G., Bondino I., McDougall S.R. (2006) Depletion of near-critical oils: Comparison between pore network model predictions and experimental results, in: International Symposium of the Society of Core Analysts held in Trondheim, Norway, 12-16 September 2006. SCA2006-32. 
11 Bondino I., McDougall S.R., Hamon G. (2005) Pore network modelling of heavy oil depressurisation: A parametric study of factors affecting critical gas saturation and 3-phase relative permeabilities, SPE J. 10, 02, 196-205. SPE-78976.

12 Bondino I., McDougall S.R., Ezeuko C.C., Hamon G. (2010) Pore-scale simulation of hysteresis effects during repressurization of gas-oil systems, SPE Annual Technical Conference and Exhibition held in Florence, Italy, 19-22 September 2010. SPE-134525.

13 Grattoni C.A., Hawes R.I., Dawe R.A. (1998) Relative permeabilities for the production of solution gas from watereflood residual oil, in: 1998 Annual Symposium of the Society of Core Analysts, SCA-9817.

14 Petersen E.B. Jr, Agaev G.S., Palatnik B., Ringen J.K., Øren P.E., Vatne K.O. (2004) Determination of critical gas saturation and relative permeabilities relevant to the depressurization of the Statfjord Field, in: International Symposium of the Society of Core Analysts held in Abu Dhabi, UAE, 5-9 October, 2004. SCA2004-33.

15 Kim T.W., Kovscek A.R. (2019) The effect of voidagedisplacement ratio on critical gas saturation, SPE J. 24, 01, 178-199.

16 Xu X., Wang J., Lv J.-P., Deng Y. (2014) Simultaneous analysis of three-dimensional percolation models, Front. Phys. 9, 1, 113-119.

17 Jan N., Stauffer D. (1998) Random site percolation in three dimensions, Int. J. Modern Phys. C 09, 02, 341-347.

18 Sykes M.F., Essam J.W. (1964) Critical percolation probabilities by series method, Phys. Rev. 133, 1A, A310-A315.

19 Van der Marck S.C. (1998) Calculation of percolation thresholds in high dimensions for FCC, BCC and diamond lattices, Int. J. Modern Phys. C 9, 4, 529-540.

20 Powell M.J. (1979) site percolation in randomly packed spheres, Phys. Rev. B 20, 10, 4194-4198.

21 Bull O., Bratteli F., Ringen J.K., Melhuus K., Bye A.L., Iversen J.E. (2011) The quest for the true residual gas saturation - an experimental approach, in: International Symposium of the Society of Core Analysts held in Austin, Texas, USA, 18-21 September, 2011. SCA2011-03.

22 Cense A., Reed J., Egermann P. (2016) SCAL for gas reservoirs: A contribution for better experiments, in: International Symposium of the Society of Core Analysts held in Snowmass, Colorado, USA, 21-26 August 2016. Paper SCA2016-023.

23 Satik C., Robertson C., Kalpacki B., Gupta D. (2004) A study of heavy oil solution gas drive for Hamaca Field: Depletion studies and interpretations, in: SPE Paper 86967 presented at the SPE International Thermal Operations and Heavy Oil Symposium and Western Regional Meeting, Bakersfield, CA, 16-18, March.

24 Lenormand R., Touboul E., Zarcone C. (1988) Numerical models and experiments on immiscible displacements in porous media, J. Fluid Mech. 189, 165-187.

25 Berg S., Oedai S., Landman A.J., Brussee N., Boele M., Valdez R., van Gelder K. (2010) Miscible displacement of oils by carbon disulfide in porous media: Experiments and analysis, Phys. Fluids 22, 113102.

26 Berg S., Ott H. (2012) Stability of $\mathrm{CO}_{2}$-brine immiscible displacement, Int. J. Greenhouse Gas Control 11, 188-203.
27 Ji W., Dahmani A., Ahlfeld D.P., Lin J.D., Hill E. III (1993) Laboratory study of air sparging: Air flow visualization, Groundwater Monit. Remediat. 13, 4, 115-126.

28 Plummer C.R., Nelson J.D., Zumwalt G.S. (1997) Horizontal and vertical well comparison for in-situ air sparging, Ground Water Monit. Rev. 17, 1, 91-96.

29 Mumford K.G. (2008) Spontaneous expansion and mobilization of gas above DNAPL, PhD Thesis, McMaster University.

30 Zou S., Armstrong R.T., Arns J.Y., Arns C.H., Hussain F. (2018) Experimental and theoretical evidence for increased ganglion dynamics during fractional flow in mixed-wet porous media, Water Resour. Res. 54, 5, 3277-3289.

31 Armstrong R.T., McClure J.E., Berrill M.A., Rücker M., Schlüter S., Berg S. (2016) Beyond Darcy's law: The role of phase topology and ganglion dynamics for two fluid flow, Phys. Rev. E 94, 043113.

32 Berg S., Gao Y., Georgiadis A., Brussee N., Coorn A., van der Linde H., Dietderich J., Alpak F.O., Eriksen D., Mooijervan den Heuvel M., Southwick J., Appel M., Wilson O.B. (2019) Determination of critical gas saturation by micro-CT, in: 2019 Annual Symposium of the Society of Core Analysts, Aug 25-29, Pau, France, Paper SCA2019-021.

33 Berg S., Gao Y., Georgiadis A., Brussee N., Coorn A., van der Linde H., Dietderich J., Alpak F.O., Eriksen D., Mooijervan den Heuvel M., Southwick J., Appel M., Wilson O.B. (2020) Determination of critical gas saturation by micro-CT, Petrophysics 61, 2, 133-150.

34 van Wageningen W.F.C., Maas J.G. (2007) Reservoir simulation and interpretation of the RECOPOL ECMB pilot in Poland, in: 2007 International Coalbed Methane Symposium, Paper 07\%.

35 Garfi G., John C.M., Lin Q., Berg S., Krevor S. (2020) Fluid surface coverage showing the controls of rock mineralogy on the wetting state, Geophys. Res. Lett. 47, 8, e2019GL086380.

36 Buades A., Coll B., Morel J.-M. (2005) A non-local algorithm for image denoising, in: 2005 IEEE Computer Society Conference on Computer Vision and Pattern Recognition (CVPR'05), 2, pp. 60-65.

37 Alpak F.O., Gray F., Saxena N., Dietderich J., Hofmann R., Berg S. (2018) A distributed parallel multiple-relaxationtime lattice Boltzmann method on graphic processing units for the rapid and scalable computation of absolute permeability from high-resolution 3D micro-CT images, Comput. Geosci. 22, 3, 815-832.

38 Alpak F.O., Zacharoudiou I., Berg S., Dietderich J., Saxena N. (2019) Direct simulation of pore-scale two-phase visco-capillary flow on large digital rock images using a phase-field lattice Boltzmann method on general-purpose graphics processing units, Comput. Geosci. 23, 5, 849-880.

39 Bhatnagar P.L., Gross E.P., Krook M. (1954) A model for collision processes in gases. I. Small amplitude processes in charged and neutral one-component systems, Phys. Rev. 94, 511-525.

40 D'Humières D., Ginzburg I., Krafcyzk M., Lallemand P., Luo L.-S. (2002) Multiple-relaxation-time lattice Boltzmann models in three dimensions, Philos. Trans. R. Soc. A. 360, 437-451.

41 Roof J.G. (1970) Snap-off of oil droplets in water-wet pores, SPE J. 10, 1, 85-90. 
42 Berg S., Armstrong R.T., Ott H., Georgiadis A., Klapp S., Schwing A., Neiteler R., Brussee N., Makurat A., Leu L., Enzmann F., Schwarz J.-O., Wolf M., Khan F., Kersten M., Irvine S., Stampanoni M. (2014) Multiphase flow in porous rock imaged under dynamic flow conditions with fast X-ray computed microtomography, Petrophysics 55, 4, 304-312.

43 Evseev N., Armstrong R.T., Berg S., Dinariev O., Klemin D., Koroteev D., Safonov S. (2016) Modeling of pore-scale twophase flow phenomena using density functional hydrodynamics, Transp. Porous Media 112, 3, 577-607.

44 Rücker M., Berg S., Armstrong R.T., Georgiadis A., Ott H., Schwing A., Neiteler R., Brussee N., Makurat A., Leu L., Wolf M., Khan F., Enzmann F., Kersten M. (2015) From connected pathway flow to ganglion dynamics, Geophys. Res. Lett. 42, 3888-3894.

45 Reamer H.H., Opfell J.B., Sage B.H. (1956) Diffusion coefficients in hydrocarbon systems, methane-decanemethane in liquid phase, Ind. Eng. Chem. 48, 2, 275-282.

46 Bartels W.-B., Rücker M., Berg S., Mahani H., Georgiadis A., Fadili A., Brussee N., Coorn A., van der Linde H., Hinz C., Jacob A., Wagner C., Henkel S., Enzmann F., Bonnin A., Stampanoni M., Ott H., Blunt M., Hassanizadeh M. (2017) Fast X-ray micro-CT study of the impact of brine salinity on the pore-scale fluid distribution during waterflooding, Petrophysics 58, 1, 36-47.

47 Maheshwari S., van Kruijsdijk C., Sanyal S., Harvey A.D. (2020) Nucleation and growth of a nanobubble on rough surfaces, Langmuir 36, 4108-4415.

48 Bultreys T., Sing K., Raeini A.Q., Ruspini L.C., Oren P.-E., Berg S., Rücker M., Bijeljic B., Blunt M.J. (2019) Verifying pore network models of imbibition in rocks using timeresolved synchrotron imaging, Water Resour. Res. 56, 6, e2019WR026587.

49 Xu K., Bonnecaze R., Balhoff M. (2017) Egalitarianism among bubbles in porous media: An Ostwald ripening derived anticoarsening phenomenon, Phys. Rev. Lett. 119, 264502.

50 de Chalendar J.A., Garing C., Benson S.M. (2018) Pore-scale modelling of Ostwald ripening, J. Fluid Mech. 835, 363-392.

51 Xu K., Mehmani Y., Shang L., Xiong Q. (2019) Gravityinduced bubble ripening in porous media and its impact on capillary trapping stability, Geophys. Res. Lett. 46, 13, 804-813.

52 Herring A.L., Andersson L., Schlüter S., Sheppard A., Wildenschild D. (2015) Efficiently engineering pore-scale processes: the role of force dominance and topology during nonwetting phase trapping in porous media, Adv. Water Resour. 79, 91.

53 McClure J.E., Armstrong R.T., Berrill M.A., Schlüter S., Berg S., Gray W.G., Miller C.T. (2018) A geometric state function for two-fluid flow in porous media, Phys. Rev. Fluids 3, 8, 084306.

54 Armstrong R.T., McClure J.E., Robins V., Liu Z., Arns C.H., Schlüter S., Berg S. (2019) Porous media characterization using Minkowski functionals: Theories, applications and future directions, Transp. Porous Media 130, 305-335. https://doi.org/10.1007/s11242-018-1201-4.

55 Schlüter S., Berg S., Rücker M., Armstrong R.T., Vogel H.-J., Hilfer R., Wildenschild D. (2016) Pore scale displacement mechanisms as a source of hysteresis for two-phase flow in porous media, Water Resour. Res. 52, 3, 2194-2205.

56 Alzayer A., Voskov D.V., Tchelepi H.A. (2018) Relative permeability of near-miscible fluids in compositional simulators, Trans. Porous Med. 122, 3, 547-573.

57 Berg S., Rücker M., Ott H., Georgiadis A., van der Linde H., Enzmann F., Kersten M., Armstrong R.T., de With S., Becker J., Wiegmann A. (2016) Connected pathway relative permeability from pore scale imaging of imbibition, $A d v$. Water Resour. 90, 24-35.

58 Liu Z., Herring A., Sheppard A., Arns C., Berg S., Armstrong R.T. (2017) Morphological characterization of two-phase flow using X-ray microcomputed tomography flow-experiments, Transp. Porous Media 118, 1, 99-117.

59 Rücker M., Bartels W.-B., Garfi G., Shams M., Bultreys T., Boone M., Pieterse S., Maitland G.C., Krevor S., Cnudde V. Mahani H., Berg S., Georgiadis A., Luckham P.F. (2020) Relationship between wetting and capillary pressure in a crude oil/brine/rock system: From nano-scale to core-scale, J. Colloid Interface Sci. 562, 7, 159-169.

60 Lin Q., Bijeljic B., Krevor S.C., Blunt M.J., Rücker M., Berg S., Coorn A., van der Linde H., Georgiadis A., Wilson O.B. (2019) A new waterflood initialization protocol for pore-scale multiphase flow experiments, Petrophysics 60, 02, 264-272.

61 Vachaparambil K.J., Einarsrud K.E. (2018) Explanation of bubble nucleation mechanisms: A gradient theory approach, J. Electrochem. Soc. 165, 10, E504-E512.

62 Fletcher N.H. (1958) Size effect in heterogeneous nucleation, J. Chem. Phys. 29, 572.

63 Thanh N.T.K., Maclean N., Mahiddine S. (2014) Mechanisms of nucleation and growth of nanoparticles in solution, Chem. Rev. 114, 15, 7610-7630.

64 Aursand P., Gjennestad M.Aa, Aursand E., Hammer M., Wilhelmsen $\varnothing$. (2017) The spinodal of a single and multicomponent fluids and its role in the development of modern equations of state, Fluid Phase Equilib. 436, 98-112.

65 Liger-Belair G., Marchal R., Jeandet P. (2002) Close-up on bubble nucleation in a glass of champagne, Am. J. Enol. Vitic. 53, 2, 151-153.

66 Gomis A.M., Reyes-Labarta J.A., Cayuelas M.D.S., del Mar Olaya Lopez M. (2011) GE models and algorithms for condensed phase equilibrium data regression in ternary systems: Limitations and proposals, Open Thermodyn. J. 5, Suppl 1-M4, 48-62.

67 Marcilla A., Serrano M.D., Reyes-Labarta J.A., Olaya M.M. (2012) Checking liquid-liquid pait point conditions and their application in ternary systems, Ind. Eng. Chem. Res. 51, 5098-51-5098-52.

68 Koroteev D., Dinariev O., Evseev N., Klemin D., Safonov S., Gurpinar O., Berg S., van Kruijsdijk C., Myers M., Hathon L., de Jong H., Armstrong R. (2013) Application of digital rock technology for chemical EOR screening, in: $S P E$ Enhanced Oil Recovery Conference held in Kuala Lumpur, Malaysia, 2-4 July 2013. SPE-165258.

69 Dinariev O.Y., Evseev N.E. (2008) Filtration of a gascondensate mixture near the hydraulic fracture, J. Eng. Phys. Thermophys. 81, 3, 429-437. 